\title{
ON THE CONSTRUCTION OF BRANCHED COVERINGS OF LOW-DIMENSIONAL MANIFOLDS
}

\author{
BY \\ ISRAEL BERSTEIN $^{1}$ AND ALLAN L. EDMONDS ${ }^{1}$
}

\begin{abstract}
Several general results are proved concerning the existence and uniqueness of various branched coverings of manifolds in dimensions 2 and 3. The results are applied to give a rather complete account as to which 3-manifolds are branched coverings of $S^{3}, S^{2} \times S^{1}, P^{2} \times S^{1}$, or the nontrivial $S^{3}$-bundle over $S^{1}$, and which degrees can be achieved in each case. In particular, it is shown that any closed nonorientable 3-manifold is a branched covering of $P^{2} \times S^{1}$ of degree which can be chosen to be at most 6 and with branch set a simple closed curve. This result is applied to show that a closed nonorientable 3-manifold admits an open book decomposition which is induced from such a decomposition of $P^{2} \times S^{1}$.
\end{abstract}

Introduction. A well-known classical theorem of J. W. Alexander [A1] states that a closed orientable piecewise linear $n$-manifold can be expressed as a branched covering of the $n$-sphere $S^{n}$. S. Stoilow [Sto, pp. 77, 119] and M. Heins [He] subsequently gave separate proofs in the 2-dimensional case. The result is of particular interest in dimension three, where it has been refined by H. M. Hilden [Hil], U. Hirsch [Hir1], on and J. M. Montesinos [M]. One of the applications of Alexander's theorem in dimension three was his proof [A2] that every closed orientable 3-manifold admits what is now called an open book decomposition.

In this paper a number of general results are proved concerning the existence and uniqueness of various branched coverings in dimensions two and three. These results enable us to give a rather complete account as to which 3-manifolds are branched coverings of $S^{3}, S^{2} \times S^{1}, P^{2} \times S^{1}$, or the nontrivial $S^{2}$-bundle over $S^{1}$, and which degrees can be achieved in each case. In particular it is shown that any nonorientable 3-manifold is a branched covering of $P^{2} \times S^{1}$ and that nonorientable 3-manifolds also admit open book decompositions which are induced from such a decomposition $P^{2} \times S^{1}$.

Received by the editors June 6, 1977.

AMS (MOS) subject classifications (1970). Primary 55A10; Secondary 57A10, 55A25, 57A05, 57A65.

Key words and phrases. Branched covering, 3-manifold, 2-manifold, nonorientable 3 manifold, open book decomposition, classical knot.

'Supported in part by a National Science Foundation grant.

(C) 1979 American Mathematical Society 0002-9947/79/0000-0003/\$10.50 
We have tried to formulate our techniques used in proving these results in such a way that they should be useful for subsequent applications as well.

After providing in $\$ 1$ some background material and in $\$ 2$ a discussion of the classification of general branched coverings in dimension two due to $\mathrm{A}$. Hurwitz [Hur], we focus in $\$ \S 3-4$ on a particular class of "simple" branched coverings-branched coverings of degree $n$, each of whose point inverses contains at least $n-1$ points, and therefore at most one singular point. It turns out that in dimensions two and three such branched coverings are generic among branched coverings. That is, they form an open and dense set in the space of all branched coverings between two given manifolds (Propositions (3.1) and (3.3) and Theorem (6.5)). See also [Hir3]. Therefore results about simple branched coverings often imply results about general branched coverings.

One of the most important facts about simple branched coverings over the 2-sphere $S^{2}$ is that they are unique up to equivalence, for each degree (Theorem (3.4)). This result, in somewhat different form, actually goes back over one hundred years to Lüroth [Lu]. An alternative topological proof has recently been given by Hirsch [Hir2].

These simple branched coverings $M^{2} \rightarrow S^{2}$ also generalize the particular branched coverings of degree 3 constructed by Hilden [Hil] and Hirsch [Hir1] and enjoy similar properties:

(i) Any homeomorphism of $M^{2}$ is isotopic to one which covers a homeomorphism of $S^{2}$ (Theorem (4.1)). Hirsch [Hir2] indicates that his results imply this for orientation-preserving homeomorphisms.

(ii) There is a homeomorphism of $\boldsymbol{M}^{2}$ which is isotopic to the identity and covers a homeomorphism of $S^{2}$ which induces any prescribed permutation of the branch set (Theorem (4.9)).

We show in $\$ 5$ that any map of a surface of genus $g$ to a surface of genus 1 is homotopic to a branched covering provided it has degree $n \geqslant 2, g \geqslant$ $\max \{3, n\}$, and the map is surjective on fundamental groups (Theorem (5.1)). It is an interesting problem to characterize in general what homotopy classes of maps between manifolds contain branched coverings. (Added in proof. See the abstract of the second author, Deforming maps to branched coverings, Notices Amer. Math. Soc. 25 (1978), A-260.)

Next in $\$ 6$ we make the transition to the study of 3-manifolds. Here we prove various extension theorems typified by the assertion that any branched covering $\partial M^{3} \rightarrow S^{2}$ of degree at least 3 extends to a branched covering $M^{3} \rightarrow D^{3}$ (Corollary (6.3)). In this section we also show that any branched covering $M^{3} \rightarrow N^{3}$ can be adjusted to have the branch set be a 1-manifold (Corollary (6.6)). Most of our constructed branched coverings have this property to start with.

In $\$ \S 7-10$ we present applications of the preceding existence, uniqueness, 
and extension results. In $\$ 7$ we show that a closed orientable 3-manifold $M^{3}$ is a branched covering of $S^{3}$ of any desired degree greater than 2 (Theorem (7.1)), and that $M^{3}$ is a branched covering of $S^{2} \times S^{1}$ if and only if $H^{1}\left(M^{3}\right.$; Z) $\neq 0$ (Theorem (7.3)). Also the number of components of the branch set in either case can be any number greater than 0 .

In $\$ 8$ we construct branched coverings of closed nonorientable 3-manifolds. If $M^{3}$ is nonorientable but $w_{1}\left(M^{3}\right)$ is integral, then $M^{3}$ can be cut open along an orientable surface to yield an orientable 3-manifold and $M^{3}$ can be expressed as a branched covering of $S^{2} \otimes S^{1}$, the nontrivial $S^{2}$-bundle over $S^{1}$ (Theorem (8.3)). If $w_{1}\left(M^{3}\right)$ is not integral then $M^{3}$ can be expressed as the union of an orientable 3-manifold and a nonorientable bundle over an orientable 2-manifold (Proposition (8.5)). This decomposition then leads to branched coverings of $M^{3}$ over $P^{2} \times S^{1}$, the simplest 3-manifold of this sort (Theorem (8.8)). The possible degrees depend on more subtle properties of $H^{*}\left(M^{3}\right)$. Since $S^{2} \otimes S^{1}$ is a 2 -fold covering of $P^{2} \times S^{1}$, this shows that every closed nonorientable 3-manifold is a branched covering of $P^{2} \times S^{1}$.

In $\$ 9$ we apply the preceding result to show that every closed nonorientable 3-manifold admits an open book decomposition (Theorem (9.8)). The first step is to show that $P^{2} \times S^{1}$ admits such a decomposition (Theorem (9.1)). We then show how to pull back this decomposition through a branched covering to deduce the desired result, in much the same way Alexander [A2] did for orientable manifolds which are branched coverings of $S^{3}$ (Theorem (9.3) and Proposition (9.7)). These results answer questions raised by $F$. González-Acuña. $^{2}$

As a final application of the earlier results we show in $\$ 10$ that any knot in $S^{3}$ can be obtained as the inverse image of the trivial knot under a branched covering $S^{3} \rightarrow S^{3}$, with branch set in the complement of the trivial knot (Theorem (10.1)). This answers a question raised by D. Goldsmith.

In a concluding appendix we discuss the nonorientable analogue of the higher-dimensional Alexander branched covering theorem. We show that any $2 n$-dimensional closed nonorientable manifold is a branched covering of real projective space $P^{2 n}$, but that there can be no such universal target in the $(2 n+1)$-dimensional case if $n \geqslant 2$.

Except for our use of Lickorish's construction of a finite set of generators for the homeotopy group of a closed orientable 2-manifold [Li2] and of Stallings' fibration theorem [Sta], we have attempted to make this paper essentially self-contained.

We thank George Francis for drawing the figures for this paper.

\footnotetext{
${ }^{2}$ Gonzalez-Acuña has recently informed us that he also has proved the existence of open book decompositions using other techniques.
} 
1. Preliminaries. Throughout we shall work in the piecewise linear (PL) category. Hudson [Hud] is a convenient reference for basic facts and standard notation and definitions. With reasonable care most of our results can be formulated and proved in either the differentiable or topological categories. All manifolds will be compact and connected with boundary allowed unless explicitly stated otherwise. All maps $f: M^{m} \rightarrow N^{m}$ between $m$-manifolds will be $P L$ and proper, that is, $f^{-1}\left(\partial N^{m}\right)=\partial M^{m}$.

Some standard notation will be used throughout: $D^{m}$ will denote a standard PL $m$-ball, $S^{m-1}$ will denote its boundary $\partial D^{m}$, and $P^{m}$ will denote real projective $m$-space.

A (proper, PL) map $\varphi: M^{m} \rightarrow N^{m}$ between manifolds is called a branched covering if it is finite-to-one and open. In practice this is checked using the equivalent condition that the set of points of $M^{m}$ at which $\varphi$ fails to be a local homeomorphism, the singular set, is a subpolyhedron of $M^{m}$ of codimension 2. The image in $N^{m}$ of the singular set of the branched covering $\varphi$ is called the branch set of $\varphi$ and denoted by $B_{\varphi}$, or simply by $B$ if no confusion arises. The branch cover is $\varphi^{-1}(B)$. The fibers of $\varphi$ are the finite sets $\varphi^{-1}(y)$, $y \in N^{m}$. A fiber $\varphi^{-1}(y)$ is principal if $y \in N-B_{\varphi}$.

Two branched coverings $\varphi_{0}, \varphi_{1}: M^{m} \rightarrow N^{m}$ are equivalent if there exist homeomorphisms $f: M^{m} \rightarrow M^{m}$ and $g: N^{m} \rightarrow N^{m}$ such that $g \varphi_{0}=\varphi_{1} f$, and are b-homotopic if there is a homotopy $\theta_{t}: M^{m} \rightarrow N^{m}, 0 \leqslant t \leqslant 1$, such that $\theta_{0}=\varphi_{0}, \theta_{1}=\varphi_{1}$, and each $\theta_{t}$ is a branched covering.

Note the analogue for branched coverings of the "Alexander trick": Any branched covering $\varphi: D^{m} \rightarrow D^{m}$ is $b$-homotopic to the cone $C\left(\varphi \mid \partial D^{m}\right)$, and, hence, two such branched coverings which agree on $\partial D^{m}$ are $b$-homotopic.

Suppose that $\varphi: M^{m} \rightarrow N^{m}$ is a branched covering and that $N^{m}$ is orientable. Then an orientation of $N^{m}$ determines an orientation of $M^{m}$ such that $\varphi$ is orientation-preserving: simply triangulate $M^{m}$ and $N^{m}$ so that $\varphi$ is simplicial and orient the $m$-simplices of $M^{m}$ so that $\varphi$ is orientation-preserving on each simplex. A branched covering $\varphi: M^{m} \rightarrow N^{m}$ which preserves specified orientations of $M^{m}$ and $N^{m}$ will be called an oriented branched covering.

More generally, one easily sees that the branched covering $\varphi: M^{m} \rightarrow N^{m}$ maps orientation-reversing loops in $M^{m}$ to orientation-reversing loops in $N^{m}$. Therefore, if $w_{1}\left(M^{m}\right) \in H^{1}\left(M^{m} ; \mathbf{Z} / 2\right) \approx \operatorname{Hom}\left(H_{1}\left(M^{m}\right) ; \mathbf{Z} / 2\right)$ denotes the first Stiefel-Whitney class of $M^{m}$, which assigns 1 to each orientationreversing loop in $M^{m}$, then $\varphi^{*} w_{1}\left(N^{m}\right)=w_{1}\left(M^{m}\right)$.

If $\varphi: M^{m} \rightarrow N^{m}$ is a branched covering, the degree of $\varphi, \operatorname{deg}(\varphi)$, is the maximum cardinality of a fiber. If $\varphi$ is only a map we shall mean by $\operatorname{deg}(\varphi)$ the absolute degree of $\varphi$ in the sense of Epstein [E]. In case the map $\varphi$ preserves $w_{1}$, then $\varphi$ lifts to a map $\tilde{\varphi}: \tilde{M}^{m} \rightarrow \tilde{N}^{m}$ of orientable double covers 
and $\operatorname{deg}(\varphi)=\operatorname{deg}(\tilde{\varphi})$, and the latter is the ordinary homological degree up to sign. It is then easy to see that if $\varphi$ is a branched covering the two definitions of $\operatorname{deg}(\varphi)$ coincide.

A map $f: X \rightarrow Y$ is called primitive if $f_{*}: \pi_{1}(X, *) \rightarrow \pi_{1}(Y, *)$ is surjective. In particular, a primitive branched covering is one which has no unbranched covering as a left factor. Similarly a cohomology class $v \in H^{1}(X ; Z)$ is primitive (or indivisible) if a map $X \rightarrow S^{1}$ representing $v$ is primitive.

2. Classification of branched coverings in dimension two. We now turn to the classical description of branched coverings of 2-manifolds. We wish to thank George Francis for providing us at a timely moment with copies of his work which provides a nice introduction to the original sources in this subject.

Let $\varphi: M^{2} \rightarrow N^{2}$ be a branched covering of degree $n$. Near any point $x \in M^{2}, \varphi$ is equivalent to the complex function $z \mapsto z^{d}$ for some $d \geqslant 1$ and we define the local degree of $\varphi$ at $x, \operatorname{deg}(\varphi ; x)=d$. Then the RiemannHurwitz formula states that

$$
\chi\left(M^{2}\right)=n \chi\left(N^{2}\right)-\Sigma(\operatorname{deg}(\varphi ; x)-1)
$$

where $\chi\left(M^{2}\right)$ denotes the Euler characteristic of $M^{2}$ and the summation extends over all $x$ in the singular set of $\varphi$. The classification theorem for 2-manifolds thus allows one to determine $M^{2}$, given $n, N^{2}$, and some information about the branch set.

In [Hur] A. Hurwitz showed how to associate to any branched covering $\varphi$ : $M^{2} \rightarrow N^{2}$ of degree $n$ certain data which we shall call a Hurwitz system for $\varphi$. In more modern terminology, the branched covering $\varphi$ is determined by the associated unbranched covering $\varphi_{0}: M^{2}-\varphi^{-1}(B) \rightarrow N^{2}-B$ by passage to end point compactifications. And the unbranched covering $\varphi_{0}$ is determined by a homomorphism

$$
\rho(\varphi): \pi_{1}\left(N^{2}-B, *\right) \rightarrow \delta_{n}
$$

where $\delta_{n}$ denotes the symmetric group on $n$ letters and $* \in N^{2}-B$ is a base point. The representation $\rho$ is determined modulo inner automorphisms of $\mathcal{S}_{n}$ by choosing a one-to-one correspondence $\varphi^{-1}(*) \leftrightarrow\{1,2, \ldots, n\}$ and assigning to a loop $\alpha$ in $N^{2}-B$ based at $*$ the permutation of $\{1,2, \ldots, n\}$ induced by transporting $\varphi^{-1}(*)$ around $\alpha$.

(2.1) Hurwitz Classification Theorem. Two branched coverings $\varphi, \psi$ : $M_{i}^{2} \rightarrow N^{2}, i=0,1$, are equivalent if and only if there is a homeomorphism $h$ : $\left(N^{2}, B_{\varphi}, *\right) \rightarrow\left(N^{2}, B_{\psi}, *\right)$ such that $\rho(\varphi)=\rho(\psi) h_{*}$ modulo inner automorphisms of $\delta_{n}$.

(2.2) HURWITZ EXISTENCE TheOReM. For any finite set $B \subset N^{2}$ and representation $\rho$ : $\pi_{1}\left(N^{2}-B, *\right) \rightarrow \delta_{n}$ there is a degree $n$ branched covering $\varphi$ : $M^{2} \rightarrow N^{2}$, where $M^{2}$ is perhaps not connected, with $B_{\varphi} \subset B$ and $\rho(\varphi)=\rho$. 
To formulate (2.2) more nearly in the spirit of Hurwitz and in the way in which we shall use it, we shall restrict attention to the case $N^{2}=S^{2}$.

Fix an orientation of $S^{2}$. Let $B \subset S^{1}$ be a finite set of cardinality $k$, and let $* \in S^{2}-B$ be a base point. Choose a simple system of rays $r_{1}, r_{2}, \ldots, r_{k}$ connecting * to each point of $B$, indexed in such a way that a small circle about * oriented by $S^{2}$ meets the $r_{i}$ 's in order of increasing subscript (modulo k).

The choice of orientation and $\left\{r_{i}\right\}$ identifies $\pi_{1}\left(S^{2}-B, *\right)$ with the free group on $k-1$ generators $x_{1}, x_{2}, \ldots, x_{k-1}$, where $x_{i}$ corresponds to a loop which runs out along $r_{i}$, goes around the end point in the direction determined by the orientation, and returns along $r_{i}$. Or, better for our purposes, this identifies $\pi_{1}\left(S^{2}-B, *\right)$ with the free group on $k$ generators $x_{1}$, $x_{2}, \ldots, x_{k}$ modulo the relation $x_{1} x_{2} \cdots x_{k}=1$. A representation $\rho: \pi_{1}\left(S^{2}\right.$ $-B, *) \rightarrow \delta_{n}$ then determines, and is determined by, an ordered $k$-tuple $\left(\alpha_{1}, \alpha_{2}, \ldots, \alpha_{k}\right)$ of permutations of $\delta_{n}$ subject to the condition that $\alpha_{1} \alpha_{2} \cdots \alpha_{k}=(1)$ in $\delta_{n}$.

Then (2.2) says that for any $k$-tuple $\left(\alpha_{1}, \alpha_{2}, \ldots, \alpha_{k}\right)$ of nontrivial elements in $\delta_{n}$ there is a branched covering $\varphi: M^{2} \rightarrow S^{2}$ with branch set $B$ of cardinality $k, \operatorname{deg}(\varphi)=n$, and representation $\rho: \pi_{1}\left(S^{2}-B, *\right) \rightarrow \delta_{n}$ determined as above for some choice of orientation, paths, and $\left(\alpha_{1}\right.$, $\left.\alpha_{2}, \ldots, \alpha_{k}\right)$.

Then the condition that $M^{2}$ be a connected branched covering translates into the requirement that $\rho: \pi_{1}\left(S^{2}-B, *\right) \rightarrow \mathcal{S}_{n}$ is a transitive representation, or equivalently $\left\{\alpha_{1}, \alpha_{2}, \ldots, \alpha_{k}\right\}$ generates a transitive subgroup of $\delta_{n}$.

By a Hurwitz system for $\varphi: M^{2} \rightarrow S^{2}$, then, we shall mean an orientation of $S^{2}$ together with the collections of arcs $\left\{r_{i}\right\}$ and permutations $\left\{\alpha_{i}\right\}$. However, we shall of ten suppress the $\left\{r_{i}\right\}$. For if $\left\{r_{i}^{\prime}\right\}$ is another collection of such arcs, then cutting $S^{2}$ open along the $r_{i}$ 's on the one hand and along the $r_{i}^{\prime \prime}$ s on the other hand, we obtain two trivial coverings of 2-disks together with identical gluing data given by $\left(\alpha_{1}, \alpha_{2}, \ldots, \alpha_{k}\right)$ for constructing $M^{2}$. Hence one easily constructs a homeomorphism $S^{2} \rightarrow S^{2}$ which takes one system of paths to the other and is covered by an equivalence of branched coverings.

3. Genericity and uniqueness of simple branched coverings. In this section we introduce the class of simple branched coverings. A branched covering $\varphi$ : $M^{m} \rightarrow N^{m}$ of degree $n \geqslant 2$ is simple provided that for each $y \in N^{m}$ the fiber $\varphi^{-1}(y)$ over $y$ consists of at least $n-1$ points (and hence contains at most one singular point and that of local degree 2 ).

To justify their study we begin by showing that this class is open in the set of all branched coverings between two given manifolds and in dimension two is actually dense. (A similar result is also true in dimension three but the proof is deferred until §6.) More important for our present purposes is the 
Uniqueness Theorem which goes back over 100 years to J. Lüroth [Lu] and says that a simple branched covering in dimension two is determined up to equivalence by its domain, range, and degree.

In the case of a simple branched covering $\varphi: M^{2} \rightarrow N^{2}$ in dimension 2 the Riemann-Hurwitz formula takes the simple form

$$
\chi\left(M^{2}\right)=n \chi\left(N^{2}\right)-k
$$

where $k$ is the cardinality of the branch set $B_{\varphi}$ and $n=\operatorname{deg}(\varphi)$. Similarly a Hurwitz system for $\varphi$ is especially simple because all of its permutations are just transpositions in $\mathcal{S}_{n}$.

(3.1) Proposition. Let $\varphi: M^{m} \rightarrow N^{m}$ be a simple branched covering of degree $n$ between compact manifolds, and let $\psi: M^{m} \rightarrow N^{m}$ be any other branched covering. If $\psi$ is close enough to $\varphi$ in the compact-open topology, then $\psi$ is also simple.

Proof. Let $A_{i} \subseteq$ int $B_{i} \subset B_{i}$ be $m$-disks in $N^{m}, i=1, \ldots, k$, such that $\left\{A_{i}\right\}$ covers $N^{m}$ and each $\varphi^{-1}\left(B_{i}\right)$ consists of $n$ or $n-1$ disks in $M^{m}$, each mapped surjectively onto $B_{i}$ by $\varphi$.

If $\psi$ is close enough to $\varphi$, then for each component $C$ of $\varphi^{-1}\left(B_{i}\right)$ it follows that $\psi(C) \supset A_{i}$. Hence the cardinality of any fiber of $\psi$ is at least $n-1$. If $\psi$ is close enough to $\varphi$ then $\psi$ is homotopic to $\varphi$ and so has degree $n$ also. Therefore $\psi$ is simple.

(3.2) LemMa. Any branched covering $\varphi: D^{2} \rightarrow D^{2}$ is b-homotopic rel $\partial D^{2}$ to a simple branched covering.

Proof. Let $\varphi$ have degree $n$. Then $\varphi \mid \partial D^{2}$ is equivalent to the standard $n$-fold covering. There is a simple branched covering $\psi: D^{2} \rightarrow D^{2}$ of degree $n$; for example, let $\psi$ be associated with a Hurwitz system which has $n-1$ branch points with assigned transpositions (12), (13), ., (1n). We may assume $\psi\left|\partial D^{2}=\varphi\right| \partial D^{2}$. By the "Alexander trick", $\varphi$ and $\psi$ are both $b$ homotopic to the branched covering which is the cone on $\varphi \mid \partial D^{2}$.

(3.3) Proposition. Any branched covering $\varphi: M^{2} \rightarrow N^{2}$ is b-homotopic to a simple branched covering by an arbitrarily small homotopy.

Proof. First perturb $\varphi$ slightly so that any fiber contains at most one singular point. Then choose small disk neighborhoods of the singular points in $M^{2}$ on each of which $\varphi$ defines a branched covering with image a disk. Apply (3.2) in each disk, extending trivially over the rest of $M^{2}$.

The following uniqueness theorem is the cornerstone for all our subsequent constructions of branched coverings since it implies via the Riemann-Hurwitz formula that a simple branched covering of $S^{2}$ is determined by its degree and the cardinality of its branch set. Thus in applications we shall be free to 
visualize such a simple branched covering using whatever particular model might be convenient at a given moment.

(3.4) UNIQUENESS THEOREM. If $M^{2}$ is a connected 2-manifold then any two simple branched coverings $\varphi_{0}, \varphi_{1}: M^{2} \rightarrow S^{2}$ of degree $n$ are equivalent.

This result is classical and is essentially due to Lüroth [Lu]. See also [Hur, pp. 32ff]. For the convenience of the reader we sketch our proof, which is essentially the same as the classical one. By the Hurwitz Classification Theorem of $\$ 2$ the proof reduces to the combinatorial manipulation of Hurwitz systems.

By the Riemann-Hurwitz formula and an isotopy of $S^{2}$ we may assume $\varphi_{0}$ and $\varphi_{1}$ have the same branch set $B \subset S^{2}$ and the same base point $* \in S^{2}-$ $B$. Let $\rho_{0}, \rho_{1}: \pi_{1}\left(S^{2}-B, *\right) \rightarrow \delta_{n}$ be the corresponding representations which determine $\varphi_{0}$ and $\varphi_{1}$. By the Hurwitz Classification Theorem it suffices to find a homeomorphism $f:\left(S^{2}, B, *\right) \rightarrow\left(S^{2}, B, *\right)$ such that the diagram

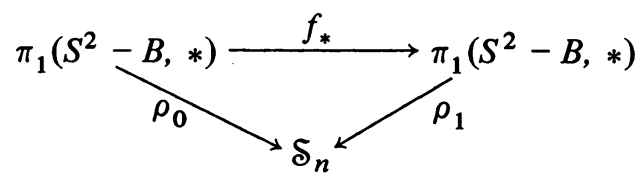

commutes.

Having chosen once and for all an orientation of $S^{2}$, then, as in \$2, a choice of paths from * to each point of $B$ allows one to describe $\rho_{0}$ (or $\rho_{1}$ ) by a sequence $\mathscr{Q}=\left(\alpha_{1}, \alpha_{2}, \ldots, \alpha_{k}\right)$ of permutations in $\delta_{n}$ such that

(i) $\alpha_{1} \alpha_{2} \cdots \alpha_{k}=(1)$;

(ii) Each $\alpha_{i}$ is a transposition ( $\varphi_{0}$ is simple);

(iii) $\left\{\alpha_{1}, \alpha_{2}, \ldots, \alpha_{k}\right\}$ generates a transitive subgroup of $\delta_{n}\left(M^{2}\right.$ is connected).

By the discussion of $\$ 2$ then it suffices to find a homeomorphism $f$ : $\left(S^{2}, B, *\right) \rightarrow\left(S^{2}, B, *\right)$ which converts (by changing the system of paths) the sequence $\mathcal{Q}$ of transpositions for $\varphi_{0}$ into that for $\varphi_{1}$, or, rather, to put the sequence $\mathcal{Q}$ into a canonical form which depends only on $n$ and $k$.

The basic move is given by the following lemma. These moves correspond to well-known generators for braid groups.

(3.5) LemMA. There is a homeomorphism $h:\left(S^{2}, B, *\right) \rightarrow\left(S^{2}, B, *\right)$ which effects the following change in $\mathbb{Q}$ :

$$
\left(\ldots, \alpha_{i-1}, \alpha_{i}, \alpha_{i+1}, \alpha_{i+2}, \ldots\right) \rightarrow\left(\ldots, \alpha_{i-1}, \alpha_{i} \alpha_{i+1} \alpha_{i}, \alpha_{i}, \alpha_{i+2}, \ldots\right) \text {. }
$$

Proof. Just twist a small disk containing only the points $b_{i}$ and $b_{i+1}$ in its interior $180^{\circ}$ in the appropriate direction, switching $b_{i}$ and $b_{i+1}$, and examine the effect on $\mathbb{Q}$. 
Operation (3.6) and its inverse do not alter properties (i)-(iii) of $\mathcal{Q}$ and can be described as saying that

any transposition $\alpha_{i}$ can be moved anywhere else in the sequence at the expense of conjugating the intervening $\alpha_{j}$ 's by $\alpha_{i}$.

Thus the Uniqueness Theorem (3.4) is reduced to the following combinatorial result.

(3.8) Proposition. Any sequence $Q=\left(\alpha_{1}, \alpha_{2}, \ldots, \alpha_{k}\right)$ in $\delta_{n}$ satisfying (i), (ii), and (iii) can be put in the canonical form

(12), (12), . ., (12), (12), (23), (23), (34), (34), . ., (n-1n), (n-1n)

using the operation (3.6) and its inverse.

Proof. Using the property that $\alpha_{1} \alpha_{2} \cdots \alpha_{k}=(1)$ one notes that $k$ is even and then inductively rearranges $\mathbb{Q}$ so that $\alpha_{2 i-1}=\alpha_{2 i}, i=1, \ldots, m$, where $k=2 m$. For suppose $\alpha_{1}=\left(x_{1} x_{2}\right)$. Some subsequent $\alpha_{i}=\left(x_{2} x_{3}\right)$ and by (3.7) we may assume it is $\alpha_{2}$. Similarly we may assume $\alpha_{3}=\left(x_{3} x_{4}\right)$, etc. Eventually we must hit some $\alpha_{r}=\left(x_{r} x_{1}\right)$. Moving $\alpha_{r-1}$ to the $r$ th position makes the new $\alpha_{r-1}=\left(x_{r-1} x_{1}\right)$. By induction on $r$ we proceed to obtain $\alpha_{2}=\left(x_{2} x_{1}\right)=$ $\left(x_{1} x_{2}\right)$. Induction on $m$ applied to $\left(\alpha_{3}, \alpha_{4}, \ldots, \alpha_{2 m}\right)$ completes the observation.

A similar argument using the transitivity of $\left\{\alpha_{1}, \alpha_{2}, \ldots, \alpha_{k}\right\}$ shows that one can arrange so that $\alpha_{1}=\alpha_{2}=(12)$. For this step it is useful to note the following two formulas which are easily derived from (3.6):

$$
(\ldots, x, x, y, \ldots) \rightarrow(\ldots, y, x, x, \ldots),
$$

that is, a pair of transpositions commutes with any other transposition; and

$$
(\ldots, x, x, y, y, \ldots) \rightarrow(\ldots y x y, y x y, y, y, \ldots) \text {, }
$$

that is, any pair of transpositions can be replaced by its conjugate by any neighboring pair and hence by any pair.

Inductively one generates pairs of (12) transpositions until the remaining transpositions are no longer transitive and the argument can be finished by induction on $k$.

4. Realization of homeomorphisms in simple branched coverings. The main results of this section are the Realization Theorem (4.1) and Theorem (4.4), which generalize the main technical results of Hilden [Hil, Theorems 4, 8]. The first says roughly that if $\varphi: M^{2} \rightarrow S^{2}$ is a simple branched covering then the group of homeomorphisms of $M^{2}$ which respect $\varphi$ surjects via the natural map onto the homeotopy group of $\boldsymbol{M}^{2}$. By the Uniqueness Theorem (3.4), Hilden proved this using his special construction when $\operatorname{deg}(\varphi)=3$, for 
orientation-preserving homeomorphisms. Also Hirsch [Hir2, p. 655] indicates that his results prove (4.1) for orientation-preserving homeomorphisms. The second says that there is a homeomorphism of $M^{2}$ which is isotopic to the identity, respects $\varphi$, and on $S^{2}$ induces any desired permutation on the branch set. Again by (3.4) Hilden proved this for $n=3$. A corollary of (4.4) is that many branched coverings $M^{3} \rightarrow N^{3}$ can be altered so as to have connected branch set.

(4.1) THEOREM. Let $M^{2}$ be a connected 2-manifold, let $\varphi: M^{2} \rightarrow S^{2}$ be a simple branched covering of degree at least 3, and let $f: M^{2} \rightarrow M^{2}$ be a homeomorphism. Then there exist homeomorphisms $h: M^{2} \rightarrow M^{2}$ and $\bar{h}: S^{2} \rightarrow$ $S^{2}$ such that $h$ is isotopic to $f$ and the diagram

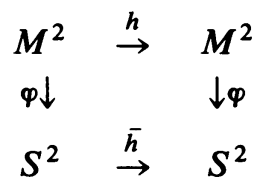

commutes.

The proof is based on lifting a large number of "twist" maps from $S^{1}$ to $M^{2}$.

Recall [Li2] that if $c$ is an oriented simple closed curve in an oriented 2-manifold $N^{2}$ then the twist map $t(c): N^{2} \rightarrow N^{2}$ is defined as follows: Let $A$ be an annular regular neighborhood of $c$ in $N^{2}$ : parametrize $A$ as $[-1,1] \times$ $\mathbf{R} / \mathbf{Z}$ so that $c$ corresponds to $0 \times \mathbf{R} / \mathbf{Z}$ and the parametrization is orientation-preserving for fixed orientations of $[-1,1] \times \mathbf{R} / \mathbf{Z}$ and of $0 \times$ $\mathbf{R} / \mathbf{Z}$. Define $t(c)$ on $A$ by $t(c)(r, \theta)=(r, \theta+r / 2+1 / 2)$ and by the identity outside of $A$. The isotopy class of $t(c)$ depends only on the isotopy class of $c$ in the oriented manifold $N^{2}$.

Similarly if $a$ is an arc in $N^{2}$ we define the disk twist $t(a): N^{2} \rightarrow N^{2}$ as follows: Let $D$ be a small disk neighborhood of $a$; outside $D$ let $t(a)$ be the identity; on $D$ let $t(a)$ twist $D 180^{\circ}$ in the direction of the orientation on $\partial D$, mapping $a$ onto itself with the ends reversed.

(4.2) Lemma. Let $\varphi: A \rightarrow D$ be a 2-fold branched covering where $A$ is an annulus, $D$ is a 2-disk, and the branch set $B=\left\{y_{1}, y_{2}\right\} \subset D^{2}$. Then the disk twist about an arc a connecting $y_{1}$ and $y_{2}$ in $D$ lifts (up to isotopy rel boundary in A) to a twist about the core of $A, t\left(\varphi^{-1}(a)\right)$.

Proof. The branched covering $\varphi$ can be realized as the orbit map for the involution $T: A \rightarrow A$ given by $T(r, \theta)=(-r,-\theta)$ and the arc $a$ to be the image of $0 \times R / Z$. Then it is easy to check that $t(0 \times \mathbf{R} / \mathbf{Z})$ commutes with $T$ and induces $t(a)$ on $D$. (Alternatively we could have used this to define disk twists, as in [Hil, §2].) 
Proof of (4.1). Let $S^{2}$ be oriented in a standard way and let $M^{2}$ be oriented so that $\varphi$ is orientation-preserving. Let $M^{2}$ have genus $g$ and let $a_{i}$ $(1 \leqslant i \leqslant g), b_{i}(1 \leqslant i \leqslant g)$, and $c_{i}(1 \leqslant i \leqslant g-1)$ denote the standard curves on $M^{2}$ pictured in Figure 4.1.

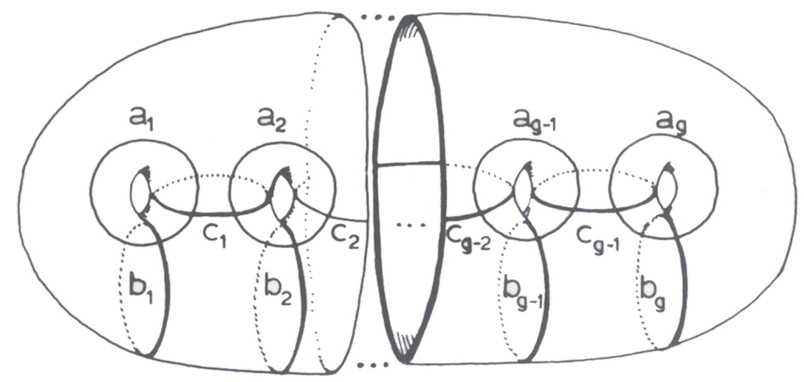

FIGURE 4.1

(Any system of curves $a_{i}, b_{i}, c_{i}$ with similar intersections would do as well.)

We shall show each of the twist maps about these $3 g-1$ curves is realized up to isotopy as in the statement of the theorem by a lift of a (disk) twist on $S^{2}$. This will complete the proof when $f$ is orientation-preserving since Lickorish has shown [Li2] that such an $f$ is isotopic to a composition of twist maps among this particular collection.

To accomplish this we use the Uniqueness Theorem (3.4) to visualize $\varphi$ in a nice way.

Let $M_{0}^{2}$ be a copy of $M^{2}$ and $\varphi_{2}: M_{0}^{2} \rightarrow S^{2}$ be the branched covering of degree 2 which is the orbit map for the involution $T$ which rotates $M_{0}^{2} 180^{\circ}$ about the horizontal axis in Figure 4.1. The Hurwitz system for $\varphi_{2}$ assigns to each of $2 g+2$ branch points the transposition (12) $\in \mathcal{S}_{2}$ and the RiemannHurwitz formula verifies that the target of $\varphi_{2}$ is indeed $S^{2}$.

Now try to prove the theorem with $\varphi_{2}$ instead of $\varphi$. It is clear that up to isotopy the curves $a_{i}$ and $c_{i}\left(1 \leqslant i \leqslant g\right.$ ) (as well as $b_{1}$ and $b_{g}$ ) can be realized within their isotopy classes as $T$-invariant simple closed curves such that each $\varphi_{2}\left(a_{i}\right)=\bar{a}_{i}$ and $\varphi_{2}\left(c_{i}\right)=\bar{c}_{i}$ is an arc in $S^{2}$. In an invariant annular neighborhood of one of these simple closed curves, $\varphi_{2}$ is equivalent to the degree 2 branched covering of Lemma (4.2). Hence the twist about such a curve can be realized as the lift of an appropriate disk twist about the corresponding arc in $S^{2}$. It is evidently impossible to realize twists about $b_{2}, \ldots, b_{g-1}$ in this way.

Now let $(n-2) S^{2}$ denote the disjoint union of $(n-2)$ copies of $S^{2}$ and extend $\varphi_{2}$ to a degree $n$ branched covering

$$
\varphi_{2}^{\prime}: M_{0}^{2} \cup(n-2) S^{2} \rightarrow S^{2}
$$

by mapping each $S^{2}$ by the identity. 
Let $\varphi_{n}^{\prime \prime}: S^{2} \rightarrow S^{2}$ be a simple branched covering of degree $n$. Its Hurwitz system assigns, without loss of generality, the transpositions

$$
\text { (12), (12), (13), (13), . . , (ln), (ln) }
$$

to the branch set.

Let

$$
\varphi_{n}:\left(M_{0}^{2} \cup(n-2) S^{2}\right) \#_{f} S^{2} \rightarrow S^{2} \# S^{2}
$$

be obtained from $\varphi_{n}^{\prime}$ and $\varphi_{n}^{\prime \prime}$ by fiber connected sum over a (12) point in each target $S^{2}$. See Figure 4.2.
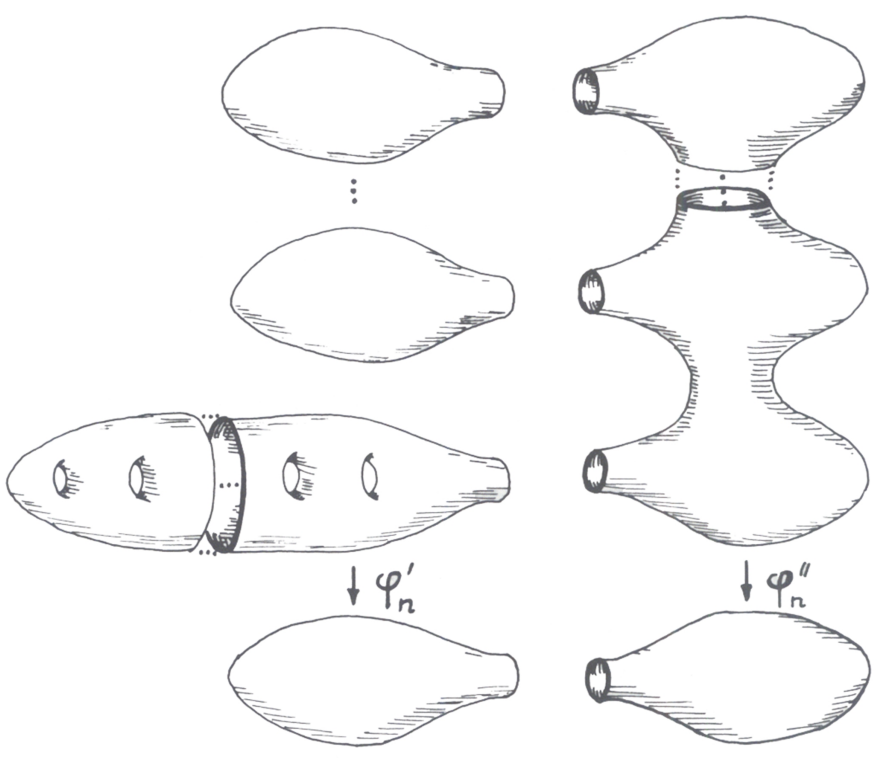

FIGURE 4.2

Since $\left(M_{0}^{2} \cup(n-2) S^{2}\right) \#_{f} S^{2} \cong M^{2}$ we may take $\varphi_{n}$ as a model for $\varphi$ by (3.4).

Now $\varphi_{n}^{-1}\left(\bar{a}_{i}\right)$ consists of the simple closed curve $a_{i}$ in $M_{0}^{2}$ near which $\varphi_{n}=\varphi_{2}$ and $(n-2)$ simple arcs mapped homeomorphically by $\varphi_{n}$. Thus the disk twist $t\left(\bar{a}_{i}\right)$ lifts to the composition of $t\left(a_{i}\right)$ and $(n-2)$ disk twists. Since disk twists are isotopic to the identity the lift of $t\left(\bar{a}_{i}\right)$ is isotopic to $t\left(a_{i}\right)$. Similarly $t\left(\bar{c}_{i}\right)$ lifts to a homeomorphism isotopic to $t\left(c_{i}\right)$.

It remains to find some arcs $\bar{b}_{i}$ in $S^{2}$ such that the disk twist $t\left(\bar{b}_{i}\right)$ lifts to a homeomorphism isotopic to $t\left(b_{i}\right)$. To this end we have depicted in Figure 4.3 the Hurwitz system for $\varphi_{n}$ with the desired arcs $\bar{b}_{i}$ (as well as $\bar{a}_{i}$ and $\bar{c}_{i}$ ) indicated. 


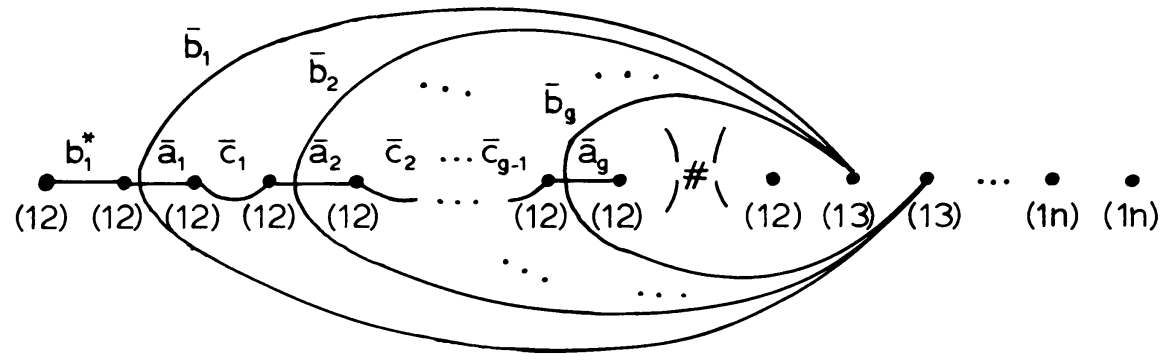

FIGURE 4.3

Consider $\bar{b}_{i}$. Over $\bar{b}_{i}$ in $M^{2}$ there are $n$ arcs $d_{i 1}, d_{i 2}, \ldots, d_{i n}$ with disjoint interiors where $d_{i 1}$ and $d_{i 3}$ have the same end points and the others are pairwise disjoint. Let $b_{i}^{\prime}$ be the loop $d_{i 1} \cup d_{i 3}$. As before the disk twist $t\left(\bar{b}_{i}\right)$ in $S^{2}$ lifts to the composition of the twist $t\left(b_{i}^{\prime}\right)$ and the disk twists $t\left(d_{i j}\right), j \neq 1,3$, which is isotopic to $t\left(b_{i}^{\prime}\right)$. It remains to show each $b_{i}^{\prime}$ is isotopic to the standard simple closed curve $b_{i}$ of Figure 4.1.

Well, $b_{i}^{\prime}$ intersects $a_{i}$ (transversely) in just one point in "sheet 1 " and misses the $a_{j}$ 's for $j \neq i$; and $b_{i}^{\prime}$ misses all the $c_{j}$ 's. Thus one easily sees that it suffices to show that although a pair $b_{i}^{\prime}$ and $b_{j}^{\prime}$ of these loops intersect in two points, they can all be isotoped apart.

If $M^{2}$ is cut open along $b_{1}, a_{1}, \ldots, a_{g}, c_{1}, \ldots, c_{g-1}$, to form $\hat{M}^{2} \cong D^{2}$, the loops $b_{1}^{\prime} b_{2}^{\prime}, \ldots, b_{g}^{\prime}$ determine simple arcs which intersect each other transversely in two points in int $\hat{M}^{2}$. Because $\hat{M}^{2}$ is a disk this means the intersection numbers $b_{i}^{\prime} \cdot b_{j}^{\prime}$ are zero and the corresponding arcs in $\hat{M}^{2}$ can be isotoped apart rel boundary in $\hat{M}^{2}$. Thus the $b_{i}^{\prime \prime}$ s can be isotoped to the standard $b_{i}$ 's.

This completes the proof of (4.1) when $f$ is orientation-preserving and it remains to realize some pair of orientation-reversing homeomorphisms as $h$ and $\bar{h}$. To do this normalize the Hurwitz system for $\varphi$ as

$$
\text { (12), (12), . . , (12), (12), (13), (13), ..., (1n), (1n), }
$$

as above. Decompose $S^{2}=D_{0}^{2} \cup D_{1}^{2}$ as the union of two disks which meet along their boundary, such that each $D_{i}^{2}$ contains half the branch points of each type: (12), .., (12), (13), ., (1n). Let $M_{i}^{2}=\varphi^{-1}\left(D_{i}^{2}\right)$ for $i=0,1$, and let $\varphi_{i}=\varphi \mid M_{i}^{2}$. Then each $M_{i}^{2}$ is connected and $\varphi_{0}$ and $\varphi_{1}$ are equivalent and $M^{2}$ is a double. A reflection in $S^{2}$ which interchanges $D_{0}^{2}$ and $D_{1}^{2}$, fixes $\partial D_{0}^{2}$, and maps branch points to like branch points, then lifts to the desired reflection in $M^{2}$.

The following lemma will be used in the proof of Theorem (4.4).

(4.3) LeMma. Let $\varphi: D^{2} \rightarrow D^{2}$ be a simple branched covering of degree 3 with branch set $\left\{y_{1}, y_{2}\right\}$ and let $t$ be a disk twist about a simple arc a connecting $y_{1}$ and $y_{2}$. Then $t^{3}$ lifts through $\varphi$ to a disk twist about the $\operatorname{arc} \varphi^{-1}(a)$. 
Proof. Let $r$ be an actual rotation of the target $D^{2}$ through $180^{\circ}$ (counterclockwise) which permutes $y_{1}=\left(\frac{1}{2}, 0\right)$ and $y_{2}=\left(-\frac{1}{2}, 0\right)$. Then $r$ lifts to a (unique since $\varphi$ is irregular and $\operatorname{deg}(\varphi)=3$ ) homeomorphism $\tilde{r}: D^{2} \rightarrow D^{2}$ which is also a rotation through $180^{\circ}$ (i.e., $\tilde{r}^{2}$ is the identity). This can be seen from the visualization of $\varphi$ in Figure 4.4.

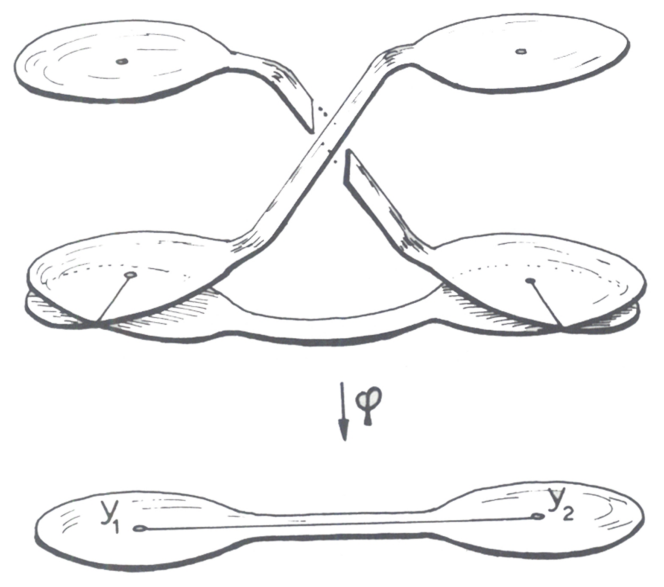

FIGURE 4.4

Now an isotopy of $r \mid \partial D^{2}$ through $-540^{\circ}$ lifts to an isotopy of $\tilde{r} \mid \partial D^{2}$ through $-180^{\circ}$ to the identity. Adjoining these isotopies to $r$ and $\tilde{r}$ in small collars in the domain and target of $\varphi$ yields the desired lifting. For in the target the resulting map on $a$ is $540^{\circ}$ ahead of the map on $\partial D^{2}$, hence corresponds to $t^{3}$; while in the domain the map on $\varphi^{-1}(a)$ is only $180^{\circ}$ ahead of the map on $\partial D^{2}($ since $\operatorname{deg}(\varphi)=3)$ and hence is an ordinary disk twist about $\varphi^{-1}(a)$.

(4.4) THEOREM. Let $\varphi: M^{2} \rightarrow S^{2}$ be a simple branched covering of degree $n$. Then there exist homeomorphisms $h: M^{2} \rightarrow M^{2}$ and $\bar{h}: S^{2} \rightarrow S^{2}$ such that the diagram

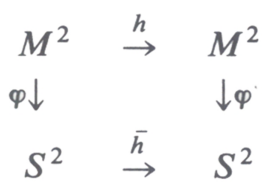

commutes, $h$ is isotopic to the identity of $M^{2}$, and $\bar{h}$ induces any prescribed permutation of the branch set $B$.

Proof. Let $B=\left\{y_{1}, y_{2}, \ldots, y_{k}\right\}$ and suppose a Hurwitz system for $\varphi$ is normalized so that the corresponding transpositions are

$$
\text { (12), (12), . . , (12), (12), (13), (13), .., (1n), (1n), }
$$

as in (3.4) and (3.8). 
It suffices to realize the transposition of $B$ which reverses $y_{i}$ and $y_{j}(i \neq j)$ and fixes the remaining branch points, since transpositions generate the symmetric group of permutations on $B$. There are two cases depending on whether the Hurwitz system assigns distinct or identical transpositions to $y_{i}$ and $y_{j}$. It suffices, then, to consider the following two special cases:

(i) $y_{i} \leftrightarrow$ (12) and $y_{j} \leftrightarrow$ (13) (distinct);

(ii) $y_{i} \leftrightarrow$ (12) and $y_{j} \leftrightarrow$ (12) (identical).

First consider case (i). Let $a$ be a simple arc from $y_{i}$ to $y_{j}$ which misses the rest of $B$. Then $\varphi^{-1}(a)$ consists of $(n-2)$ arcs, $(n-3)$ of which are mapped homeomorphically to $a$ and one of which (lying in sheets 1,2, and 3 ) is mapped in a 3-to-1 fashion onto $a$. Then, applying (4.3) to the restriction $\varphi$ to a neighborhood of this latter arc, we see that if $t(a)$ is the disk twist about $a$, then $t(a)^{3}$ lifts to a composition of disk twists in $M^{2}$ which is isotopic to the identity and permutes the fibers over $y_{i}$ and $y_{j}$ as desired.

Now consider case (ii). Choose an auxiliary point $y \in B$ which corresponds to (13) (i.e., distinct from (12)) in the Hurwitz system and choose simple arcs $a$ from $y_{i}$ to $y$ and $b$ from $y_{j}$ to $y$ such that $a$ and $b$ have disjoint interiors and miss the rest of the branch set. Then, as above, $t(a)^{3}$ and $t(b)^{3}$ lift to homeomorphisms of $M^{2}$. Therefore the homeomorphism $t(a)^{3} t(b)^{3} t(a)^{3}$ of $S^{2}$ which transposes $y_{\mathrm{i}}$ and $y_{j}$ lifts to a composition of disk twists in $M^{2}$ which is therefore isotopic to the identity, as desired.

The usefulness of (4.4) is that one can often apply it to change the number of components of the branch set of a given simple branched covering.

Let $\varphi: M^{3} \rightarrow N^{3}$ be a simple branched covering of degree $n$. Then, just as in dimension $2, \varphi$ is determined by a suitable compactification of the associated unbranched covering $\varphi_{0}: M_{0}^{3} \rightarrow N_{0}^{3}$, where $N_{0}^{3}=N^{3}-B_{\varphi}$ and $M_{0}^{3}=\varphi^{-1}\left(N_{0}^{3}\right)$. And $\varphi_{0}$ is determined by its representation $\rho: \pi_{1}\left(N_{0}^{3}, *\right) \rightarrow$ $\delta_{n}$. Define $\varphi$ to be nondegenerate if in addition $\rho$ is surjective. If $\operatorname{deg}(\varphi)=3$ or if $\pi_{1}\left(N^{3}, *\right)=0$, then $\varphi$ is fairly easily seen to be nondegenerate. Also if there is a submanifold $Q^{2} \subset N^{3}$ which meets $B_{\varphi}$ transversely and such that the representation $\pi_{1}\left(Q^{2}-B, *\right) \rightarrow \delta_{n}$, for the restriction $\varphi^{-1}(Q) \rightarrow Q$, is surjective, then $\varphi$ is also nondegenerate. This latter observation can be applied to most of the branched coverings constructed in $\$ \S 8,10$.

(4.5) Corollary. Let $M^{3}$ and $N^{3}$ be connected 3-manifolds and let $\varphi$ : $M^{3} \rightarrow N^{3}$ be a simple nondegenerate branched covering of degree $n>3$, with nonempty branch set. Then there is a simple branched covering $\psi: M^{3} \rightarrow N^{3}$ with $B_{\psi}$ connected.

Proof. Let $K$ be a one-point union of arcs emanating from $*$ in $M^{3}$, connecting * to each component of the singular set of $\varphi$. By a general position argument, we may assume that $\varphi \mid K$ is one-to-one. Let $L=\varphi(K)$. Let $V$ be 
the simplicial neighborhood of $L$ in a barycentric subdivision of a triangulation of $N^{3}$ such that $L$ and $B_{\varphi}$ are subcomplexes, and let $U=\varphi^{-1}(V)$. Let $U_{0}$ be the component of $U$ containing $K$.

We may assume that the induced simple branched covering $\varphi \mid U_{0}: U_{0} \rightarrow V$ has degree $\geqslant 3$. If it is the case that $\operatorname{deg}\left(\varphi \mid U_{0}\right)=2$ then we can change the original choice of $L$ as follows. In this case each arc of $L$ corresponds to a transposition which we may take to be (12) for each arc. Simply alter one of the arcs of $L$ by first going around a loop in $N^{3}-B_{\varphi}$ which corresponds to the transposition (23) in $\delta_{n}$. The new arc corresponds to $(23)(12)(23)^{-1}=$ (13). Thus we see $\varphi \mid U_{0}$ must have degree 3 for this new choice of $L$.

Notice also that $\varphi \mid U_{1}: U_{1} \rightarrow V$ must be a homeomorphism for any other component $U_{1}$ of $U$, since all singular points of $U$ lie in $U_{0}$ and $V \cong D^{3}$. Further, since

$$
(V, B \cap V) \cong\left(D^{2} \times I,\left(B \cap D^{2}\right) \times I\right),
$$

we see also that $\varphi^{-1}(\partial V) \cap U_{0}$ is connected.

By (4.4) we can alter, by an isotopy of $\partial U$, the way that $U$ is glued into $M^{3}$ - int $U$ in such a way as to induce a permutation of the branch points of $\partial V$, chosen so that the resulting branched covering $M^{3} \rightarrow N^{3}$ has connected branch set.

(4.6) Remark. The same kind of argument shows that if $\varphi: M^{3} \rightarrow N^{3}$ is a branched covering as in (4.5) with $B_{\varphi} \neq \varnothing$, then there is a branched covering $\psi: M^{3} \rightarrow N^{3}$ with $B_{\psi}$ consisting of arbitrarily many components.

5. Branched coverings of the torus. The results of $\$ \S 2-4$ imply immediately that any map $\varphi: M^{2} \rightarrow S^{2}$ of degree greater than or equal to 2 is homotopic to a branched covering and that two such branched coverings $\varphi_{0}$ and $\varphi_{1}$ are equivalent to $b$-homotopic branched coverings if and only if they have the same (oriented) degree-that is, they are just homotopic.

In this section we make a small start at studying analogous situations when the target manifold is more complicated. In particular we characterize a large class of mappings to the torus which are homotopic to branched coverings. The result will be used in $\$ 8$.

For $g \geqslant 0$ let $T_{g}$ the orientable surface of genus $g$.

(5.1) THEOREM. Let $f: T_{g} \rightarrow T_{1}$ be a map of degree $n \geqslant 2$ such that

(i) $g \geqslant \max \{3, n\}$ and

(ii) $n>$ index of $f_{*} \pi_{1}\left(T_{g}\right)$ in $\pi_{1}\left(T_{1}\right)$.

Then $\varphi$ is homotopic to a branched covering.

Proof. Any map $T_{g} \rightarrow T_{1}$ is determined up to homotopy by its induced homomorphism on homology since $T_{1} \cong S^{1} \times S^{1}$ has abelian fundamental group. And such a homomorphism is determined by its matrix with respect to 
the standard bases $a_{1}, a_{2}, \ldots, a_{g}, b_{1}, b_{2}, \ldots, b_{g}$ for $H_{1}\left(T_{g}: Z\right)$ and $a, b$ for $H_{1}\left(T_{1} ; \mathbf{Z}\right)$.

Also note that it suffices to prove (5.1) when $f$ is primitive. For if $f$ is not primitive $f$ factors as $p f^{\prime}$ where $f^{\prime}: T_{g} \rightarrow T_{1}$ is primitive of degree greater than one and $p$ is a covering map, so that $f$ is homotopic to a branched covering if and only if $f^{\prime}$ is.

Given these remarks the proof breaks into the following two steps.

(5.2) Proposition. There is a primitive branched covering $\psi: T_{g} \rightarrow T_{1}$ of degree $n$, provided $g \geqslant n$.

(5.3) Proposition. If $f: T_{g} \rightarrow T_{1}$ is a primitive map of degree $n$ and $g \geqslant 3$ then there are homeomorphisms $h: T_{g} \rightarrow T_{g}$ and $k: T_{1} \rightarrow T_{1}$ such that kfh has matrix

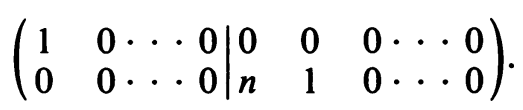

It follows then that there are homeomorphisms $h$ and $k$ such that the branched covering $k \psi h$ is homotopic to $f$.

Proof of (5.2). Let $\psi_{1}: n T_{1} \rightarrow T_{1}$ and $\psi_{2}: T_{g-n} \rightarrow T_{0}$ be branched coverings of degree $n$. Here $n T_{1}$ denotes the disjoint union of $n$ copies of $T_{1}$ each mapped homeomorphically by $\psi_{1}$ and $\psi_{2}$ is constructed by specifying a Hurwitz system with a collection of $(2 g-2) \geqslant 2(n-1)$ branch points labeled with the transitive sequence of transpositions

$$
\text { (12), (12), . . , (12), (12), (13), (13), . , (1n), (1n) }
$$

and computing the genus of the resulting total space using the RiemannHurwitz formula. Then $\psi$ is defined to be the fiber connected sum of $\psi_{1}$ and $\psi_{2}$ along a principal fiber. One easily sees that $\psi$ has the stated properties.

Before proceeding to the proof of (5.3) we prove a useful lemma of arithmetic. Let $\sim$ denote the equivalence relation on ordered pairs $(x, y)$ of integers generated by $(x, y) \sim(x, y \pm x)$ and $(x, y) \sim(x \pm y, y)$.

(5.4) LemMA. Any ordered pair of integers $(x, y)$ is equivalent to $(z, 0)$ where $z \geqslant 0$ and $z$ is the greatest common divisor (g.c.d.) of $|x|$ and $|y|$.

Proof. Notice that if $(x, y) \sim(z, w)$ then g.c.d. $\{|x|,|y|\}=$ g.c.d. $\{|z|,|w|\}$. Let $(x, y) \sim(z, w)$ where $|w|$ is as small as possible. If $w=0$ and $z \geqslant 0$ we are done; if $w=0$ and $z<0,(z, 0) \sim(z,-2 z) \sim(-z, 0)$ and again we are done.

Therefore we suppose $w \neq 0$. Then by adding multiples of $\pm w$ to $z$ if necessary we may reduce $z$ so $|z| \leqslant|w|$. But then we may add $\pm z$ to $w$, reducing $|w|$, a contradiction. 
Proof of (5.3). Consider the following automorphisms of $H_{1}\left(T_{g} ; Z\right), g>2$ (or of $H_{1}\left(T_{1} ; Z\right)$ in cases I and II):

I. $\left(a_{i}, b_{i}\right) \rightarrow\left(a_{i}, b_{i} \pm a_{i}\right)$,

II. $\left(a_{i}, b_{i}\right) \rightarrow\left(a_{i} \pm b_{i}, b_{i}\right)$,

III. $\left(a_{i}, a_{j}, b_{i}, b_{j}\right) \rightarrow\left(a_{i} \pm a_{j}, a_{j}, b_{i}, \mp b_{i}+b_{j}\right)$.

Here all unspecified generators are mapped by the identity. One easily checks that these isomorphisms preserve intersection numbers and so, as is well known, are induced by homeomorphisms of $T_{g}$. More precisely the first two are induced by appropriate twist homeomorphisms about curves representing $a_{i}$ and $b_{i}$, respectively. And the third is induced by a composition of twist homeomorphisms such as $t\left(a_{j}\right)^{-1} t\left(b_{i}\right) t(c)$, where $c$ is a curve representing $a_{j}-b_{i}$.

Now let $f_{*}$ have matrix

$$
\left(\begin{array}{l|l}
x_{1} \ldots x_{g} & y_{1} \ldots y_{g} \\
z_{1} \ldots z_{g} & w_{1} \ldots w_{g}
\end{array}\right)
$$

Then one can compute $\operatorname{deg}(f)=\sum_{i} x_{i} w_{i}-y_{i} z_{i}=n$.

Using operations I and II above we may alter $f$ by precomposing it with an appropriate homeomorphism so that by (5.4) we achieve $y_{1}=y_{2}=\cdots=y_{g}$ $=0$ (of course the other entries in the matrix will change). Then using operation III we may similarly achieve $x_{2}=x_{3}=\cdots=x_{g}=0$ and $x_{1}>0$, so that the new map, still called $f$, has a matrix of the form

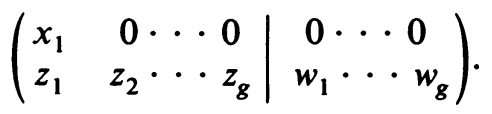

Then $x_{1}=1$ since $f_{*}$ is surjective and $w_{1}=n$ since $\operatorname{deg}(f)=x_{1} w_{1}=n$. Therefore we may assume $f_{*}$ has matrix

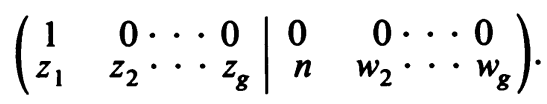

Now using operations $I$ and II in positions 2 through $g$ we kill $w_{2}, \ldots, w_{g}$ using (5.4), to obtain

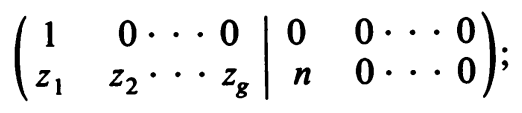

and then $z_{3}, \ldots, z_{g}$, using III, to obtain

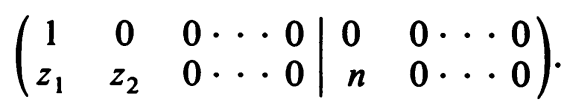

Using I and II this can be put in the form

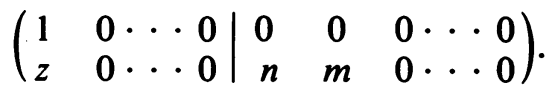


Now $f_{*}$ is surjective so that necessarily g.c.d. $\{n, m\}=1$. Then there are integers $p$ and $q$ such that $p n+q m=1$. Then since $g \geqslant 3$ by assumption we can use III to obtain

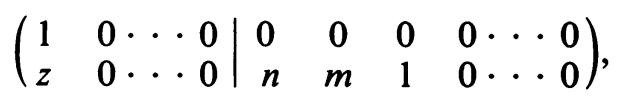

whence, by III,

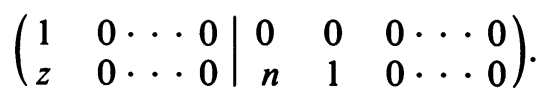

All of this has been obtained by precomposing $f$ with a homeomorphism of $T_{g}$. Finally using operation I on $T_{1}$ we obtain

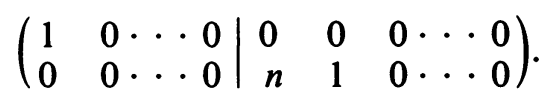

(5.5) ReMARK. By changing the end of the proof of (5.3) slightly one can show that (5.1) holds for $g=n=2$ also. (But we shall not make use of this fact.)

The branched coverings $T_{g} \rightarrow T_{1}$ constructed have the property, which we shall later use, that any simple closed curve in $T_{1}$ is isotopic to one in the complement of the branch set whose inverse image is connected. To prove this we shall discuss a broader class of branched coverings.

Define a branched covering $\varphi: M^{2} \rightarrow N^{2}$ to be local if (as in (5.2)) $M^{2}$ is connected and $\varphi$ is the fiber connected sum of an unbranched covering $\varphi_{0}$ : $M_{0}^{2} \rightarrow N^{2}$ and a branched covering $\varphi_{1}: M_{1}^{2} \rightarrow S^{2}$ where $M_{1}^{2}$ is connected. More generally define $\varphi$ to be semi-local if $\varphi$ is the fiber connected sum, along a principal fiber, of a branched covering $\varphi_{0}: M_{0}^{2} \rightarrow N^{2}$ and a branched covering $\varphi_{1}: M_{1}^{2} \rightarrow S^{2}$ where $M_{1}^{2}$ is connected. These conditions mean loosely that $\varphi$ has at least as many branch points as a simple branched covering $S^{2} \rightarrow S^{2}$ of degree $n$.

(5.6) ThEOREM. Let $\varphi: M^{2} \rightarrow N^{2}$ be a semi-local simple branched covering. Then any simple closed curve in $N^{2}$ is isotopic to a curve $c$ such that $c$ misses the branch set and $\varphi^{-1}(c)$ is connected.

Proof. First suppose $N^{2}=S^{2}$. In order that a simple closed curve $c \subset S^{2}$ $-B$ have the property that $\varphi^{-1}(c)$ is connected it is necessary and sufficient that under the representation $\rho(\varphi): \pi_{1}\left(S^{2}-B, *\right) \rightarrow \mathcal{S}_{n}, \rho(\varphi)[c]$ (well-defined only up to conjugation) be a cycle of length $n$, e.g. $(12 \cdots n)$.

Normalize the Hurwitz system for $\varphi$ to

$$
\text { (12), (12), . ., (12), (12), (13), (13), . ., (1n), (1n), }
$$

as in the Uniqueness Theorem (3.4) and (3.8). Since all simple closed curves in $S^{2}$ are isotopic we may choose $c$ to surround precisely the points assigned 
(12), (13), . , (1n). Then $c$ clearly has the desired properties.

Now consider the general case $\varphi: M^{2} \rightarrow N^{2}$ where $\varphi=\varphi_{0} \#_{f} \varphi_{1}$ as in the definition of a semilocal branched covering. Any simple closed curve $c$ in $N^{2}$ may be written up to isotopy as $c_{0} \# c_{1}$ where $c_{0} \subset N^{2}$ and $c_{1} \subset S^{2}$. As in the first case we may assume that $c_{1}$ represents an appropriate class in $\delta_{n}$, so that $c$ represents an $n$-cycle and $\varphi^{-1}(c)$ is connected.

6. Extension of branched coverings. In this section we prove various results about extending branched coverings over cobordisms. As an immediate application we sketch a proof that the analog of (3.3) holds in dimension 3: any branched covering $M^{3} \rightarrow N^{3}$ is $b$-homotopic to a simple branched covering. Further applications of these extension theorems are given in $\$ 7$ to the existence of branched coverings of orientable 3-manifolds over $S^{3}$ and over $S^{2} \times S^{1}$ of specified degree, and in $\$ 8$ to the existence of branched coverings of nonorientable 3-manifolds.

(6.1) LEMMA. Let $M^{m}$ and $N^{m}$ be connected, closed, orientable m-manifolds and let $W^{m+1}$ be an orientable cobordism obtained from $M^{m} \times I$ by adding $a$ single 1-handle $B^{1} \times D^{m}$ along $M^{m} \times 1$. Then any branched covering $\varphi$ : $M^{m} \times 0 \rightarrow N^{m} \times 0$ extends to a branched covering $\theta: W^{m+1} \rightarrow N^{m} \times I$ which is simple if $\varphi$ is.

Proof. Extend $\varphi$ to $\psi: M^{m} \times I \rightarrow N^{m} \times I$ by defining $\psi(x, t)=(\varphi(x), t)$. Let $\psi_{1}: D^{m+1} \rightarrow D^{m+1}$ be the branched covering of degree 2 which is the orbit map for the involution $\left(x_{1}, x_{2}, x_{3} \ldots x_{m+1}\right) \mapsto\left(-x_{1},-x_{2}\right.$, $\left.x_{3}, \ldots, x_{m+1}\right)$ of $D^{m+1}$. Let $\psi_{2}:(n-1) D^{m+1} \rightarrow D^{m+1}$ be the branched covering of degree $n$ obtained from $\psi_{1}$ by mapping $(n-2)$ additional $(m+1)$-disks homeomorphically.

Then there is a branched covering

$$
\theta=\psi \natural_{f} \psi_{2}: M^{m} \times I \natural_{f}(n-1) D^{m+1} \rightarrow N^{m} \times I \text { 的 } D^{m+1}
$$

obtained by forming the fiber boundary connected sum of $\psi$ and $\psi_{2}$ along principal fibers. Evidently $M^{m} \times I \natural_{f}(n-1) D^{m+1}$ is homeomorphic to $W^{m+1}$ rel $M^{m} \times 0$ and $N^{m} \times I$ 向 $D^{m+1}$ is homeomorphic to $N^{m} \times I$ rel $N^{m}$ $\times 0$ and $\theta$ is the desired extension.

(6.2) THEOREM. Let $W^{3}$ be an oriented connected cobordism between the connected 2-manifolds $M_{0}^{2}$ and $M_{1}^{2}$ and let $\varphi_{i}: M_{i}^{2} \rightarrow S^{2} \times i, i=0,1$, be oriented branched coverings of degree $n \geqslant 3$. Then there is a branched covering $\theta: W^{3} \rightarrow S^{2} \times I$ which extends $\varphi_{0}$ and $\varphi_{1}$ and which is simple if $\varphi_{0}$ and $\varphi_{1}$ are.

Proof. Choose a handle decomposition for $W^{3}$ based on $M_{0}^{2}$ having only 1-handles and 2-handles. Then we can write

$$
W^{3}=V_{0}^{3} \cup U^{2} \times I \cup V_{1}^{3}
$$


where $V_{i}^{3}$ is obtained from a collar on $M_{i}^{2}$ by adding 1-handles, for $i=0,1$, and $\partial V_{0}^{3}=M_{0}^{2} \cup U$.

By (3.3) and (6.1) $\varphi_{0}$ and $\varphi_{1}$ extend to (oriented) branched coverings $V_{0}^{3} \rightarrow S^{2} \times[0,1 / 3]$ and $V_{1}^{3} \rightarrow S^{2} \times[2 / 3,1]$ which are simple if $\varphi_{0}$ and $\varphi_{1}$ are and which are in any case simple over $S^{2} \times 1 / 3$ and $S^{2} \times 2 / 3$. The extensions determine two different oriented branched coverings of the same (oriented) degree. Therefore by the Uniqueness Theorem (3.4) and the Realization Theorem (4.1) these two are equivalent and actually $b$-homotopic. Filling in a simple $b$-homotopy $U^{2} \times I \rightarrow S^{2} \times\left[\frac{1}{3}, \frac{2}{3}\right]$ completes the proof.

(6.3) CoRollary. Let $W^{3}$ be a connected orientable 3-manifold with connected boundary and let $\varphi: \partial W^{3} \rightarrow S^{2}$ be a branched covering of degree $n \geqslant 3$. Then there is a branched covering $\theta: W^{3} \rightarrow D^{3}$ which extends $\varphi$ and is simple if $\varphi$ is.

Proof. Orient $W^{3}$ and $D^{3}$ so that $\varphi: \partial W^{3} \rightarrow S^{2}$ is oriented with respect to induced orientations. Choose a small 3-disk $B^{3} \subset$ int $W^{3}$ and let $V^{3}=W^{3}-$ int $B^{3}$. Let $\psi: B^{3} \rightarrow \frac{1}{2} D^{3}$ be any simple branched covering of degree $n$, obtained, for example, by crossing a simple branched covering $D^{2} \rightarrow D^{2}$ with I.

By (6.2) $\varphi$ and $\psi \mid \partial B^{3}$ extend to a branched covering $V^{3} \rightarrow\left(D^{3}-\right.$ int $\frac{1}{2} D^{3}$ ), which together with $\psi$ defines the desired extension.

(6.4) Remark. An analogous result holds in dimension 2: Let $M^{2}$ be an orientable, connected, 2-manifold with $\partial M^{2} \cong S^{1}$. Then any $n$-fold covering $\partial M^{2} \rightarrow S^{1}, n \geqslant 2$, extends to a simple branched covering $M^{2} \rightarrow D^{2}$. For the proof one simply constructs an appropriate Hurwitz system using the Riemann-Hurwitz formula.

We can now prove that simple branched coverings are dense in dimension 3.

(6.5) TheOREM. Any branched covering $\varphi: M^{3} \rightarrow N^{3}$ of degree $n$ is $b$ homotopic to a simple branched covering.

Proof. If $\operatorname{deg}(\varphi)=2$ then $\varphi$ is simple. So we may assume $n \geqslant 3$.

Let $Q$ denote the set of points $y \in N^{3}$ for which $\varphi^{-1}(y)$ consists of fewer than $(n-1)$ points. Evidently $Q$ is a subpolyhedron of $B_{\varphi}$ and so has dimension at most 1 . We show first how to change $\varphi$ by a $b$-homotopy so that $Q$ has dimension 0 and second how to eliminate $Q$ entirely.

We may assume that $M^{3}$ and $N^{3}$ are triangulated so that $\varphi$ is simplicial and $Q$ is a subcomplex of $N$. Let $\sigma_{1}, \ldots, \sigma_{m}$ be the 1-simplices of $Q$ with dual 2-cells $D_{1}, \ldots, D_{m}$ (subcomplexes of the first barycentric subdivision of $N^{3}$ ). Then the joins $\partial \sigma_{1} * D_{1}, \ldots, \partial \sigma_{m} * D_{m}$ are neighborhoods of int $\sigma_{1}, \ldots$, 
int $\sigma_{m}$ having disjoint interiors. Each $\varphi^{-1}\left(D_{i}\right) \rightarrow D_{i}$ is a possibly disconnected branched covering of $D_{i}$ by $(n-2)$ or fewer 2-cells, of total degree $n$. By (3.3) $\varphi \mid \varphi^{-1}\left(D_{i}\right)$ is $b$-homotopic rel $\partial \varphi^{-1}\left(D_{i}\right)$ to a simple branched covering. These $b$-homotopies together extend to $b$-homotopies over $\partial \sigma_{1} * D_{1}, \ldots, \partial \sigma_{m} * D_{m}$ using the join structures, and then over the rest of $M^{3}$ by the constant $b$-homotopy. The resulting branched covering (still call it $\varphi$ ) has $Q$ of dimension 0 .

We now suppose $Q$ is a 0 -dimensional subcomplex of $N^{3}$, say $Q=$ $\left\{v_{1}, \ldots, v_{k}\right\}$, with dual 3-cells $D_{1}, \ldots, D_{k}$. These are pairwise disjoint disk neighborhoods of $v_{1}, \ldots, v_{k}$. Each $\varphi^{-1}\left(D_{i}\right)$ consists of a number of pairwise disjoint 3-disks mapped onto $D_{i}$ by branched coverings of total degree $n$. By (6.3) and the Alexander trick for branched coverings (see §1) each $\varphi \mid \varphi^{-1}\left(D_{i}\right)$ is $b$-homotopic rel $\partial \varphi^{-1}\left(D_{i}\right)$ to a simple branched covering. These $b$-homotopies extend to all of $M^{3}$, as before, providing the desired $b$-homotopy.

(6.6) CoRollary. Any branched covering $M^{3} \rightarrow N^{3}$ is branch homotopic to one with branch set a 1-manifold.

The following result is an example of how the preceding results can be used to obtain more complicated extension theorems. It will be used in $\$ 8$.

(6.7) THEOREM. Let $W^{3}$ be a connected orientable 3-manifold with connected nonempty boundary $M^{2}$. Then a simple semilocal branched covering $\varphi: M^{2} \rightarrow$ $S^{1} \times S^{1}$ of degree $n \geqslant 3$ extends to a branched covering $\psi: W^{3} \rightarrow D^{2} \times S^{1}$ if and only if $\varphi$ extends to a map $W^{3} \rightarrow D^{2} \times S^{1}$.

Proof. Using a collar of $M^{2}$ in $W^{3}$ one sees that $\varphi$ extends to such a branched covering $\psi$ if and only if any branched covering $b$-homotopic to $\varphi$ does.

By adjusting $\varphi$ if necessary by a $b$-homotopy induced by an isotopy of $S^{1} \times S^{1}$ we may assume $S^{1} \times * \subset S^{1} \times S^{1}-B_{\varphi}$ for some base point * and $\varphi^{-1}\left(S^{1} \times *\right)$ is connected, by (5.6), since $\varphi$ is semilocal. Then $\varphi$ is automatically transverse to $S^{1} \times *$.

Let $f: W^{3} \rightarrow D^{2} \times S^{1}$ be any proper map which extends $\varphi$. By the relative transversality theorem we may assume in addition $f$ is transverse to $D^{2} \times *$. In particular $\varphi^{-1}\left(S^{1} \times *\right)$ bounds a connected two-sided 2-manifold $N^{2} \subset$ $W^{3}$ obtained as the component of $f^{-1}\left(D^{2} \times *\right)$ which contains the simple closed curve $\varphi^{-1}\left(S^{1} \times *\right)$.

In fact we may alter $f$ rel $M^{2}$ so that $N^{2}=f^{-1}\left(D^{2} \times *\right)$. For $f=\left(f_{1}, f_{2}\right)$ : $W^{3} \rightarrow D^{2} \times S^{1}$ and $f^{-1}\left(D^{2} \times *\right)=f_{2}^{-1}(*)$. Let $f_{2}^{\prime}: W^{3} \rightarrow S^{1}$ be a map which equals $f_{2}$ on a neighborhood $U$ of $N^{2} \cup M^{2}$ and maps $M^{3}-U$ into $S^{1}-*$. Now just replace $f$ by $\left(f_{1}, f_{2}^{\prime}\right)$.

By the Hurwitz Existence Theorem and the homotopy extension theorem 
we may in fact assume $f \mid N^{2}$ is a branched covering of degree $n$.

Let $\hat{W}^{3}$ denote $W^{3}$ cut open along $N^{2}$, so that $\hat{W}^{3}$ is a manifold with two copies of $N^{2}$ in $\partial \hat{W}^{3}$ and $W^{3}$ is obtained from $\hat{W}^{3}$ by suitably identifying them. Similarly $\left(D^{2} \times S^{1}\right)^{\wedge}$ denotes $D^{2} \times S^{1}$ cut open along $D^{2} \times *$. Since $f^{-1}\left(D^{2} \times *\right)=N^{2}$ there is an induced map $\hat{f}: \hat{W}^{3} \rightarrow\left(D^{2} \times S^{1}\right)^{\wedge}$ and $\hat{f} \mid \partial \hat{W}^{3}$ is a branched covering of degree $n$.

Since $\left(D^{2} \times S^{1}\right)^{1} \cong D^{3}$, it follows from (6.3) that there is a branched covering $\hat{\psi}: \hat{W}^{3} \rightarrow\left(D^{2} \times S^{1}\right)^{\wedge}$ which agrees with $\hat{f}$ on $\partial \hat{W}^{3}$. Reforming $W^{3}$ and $D^{2} \times S^{1}, \hat{\psi}$ induces the desired branched covering $\psi: W^{3} \rightarrow D^{2} \times S^{1}$.

7. Branched coverings of orientable 3-manifolds. In this section we prove a refined version of the theorems of Alexander [A1], Hilden [Hil], Hirsch [Hir1], and Montesinos $[\mathbf{M}]$ and characterize those 3-manifolds which are branched coverings of $S^{2} \times S^{1}$.

(7.1) THEOREM. Let $M^{3}$ be a closed orientable 3-manifold and $n \geqslant 3$ an integer. Then there is a simple branched covering $M^{3} \rightarrow S^{3}$ of degree $n$.

Proof. Let $\boldsymbol{M}^{3}=\boldsymbol{H}_{-} \cup \boldsymbol{H}_{+}$be a Heegard decomposition where $\boldsymbol{H}_{-}$and $H_{+}$are handlebodies identified along their boundary. Let $S^{3}=D_{-}^{3} \cup D_{+}^{3}$ where $D_{-}^{3}$ and $D_{+}^{3}$ are the upper and lower hemispheres.

By the Hurwitz Existence Theorem there is a simple branched covering $\psi$ : $\partial H_{-} \rightarrow \partial D_{-}^{3}$. By the Extension Theorem (6.3), $\psi$ extends to simple branched coverings $\varphi_{-}: H_{-} \rightarrow D_{-}^{3}$ and $\varphi_{+}: H_{+} \rightarrow D_{+}^{3}$ of degree $n$. Just set $\varphi=\varphi_{-} \cup$ $\varphi_{+} \cdot$

(7.2) REMARK. The version of (7.1) with no statement about $\boldsymbol{n}$ is that due to Alexander, while that for $n=3$ is due to Hilden, Hirsch, and Montesinos.

(7.3) THEOREM. Let $M^{3}$ be a closed orientable 3-manifold and $n>3$ any integer. Then there is a branched covering $\varphi: M^{3} \rightarrow S^{2} \times S^{1}$ of degree $n$ if and only if $H^{1}\left(M^{3} ; Z\right) \neq 0$.

Proof. First suppose there is a branched covering $\varphi: M^{3} \rightarrow S^{2} \times S^{1}$. A standard Poincaré duality argument shows that $\varphi^{*}: H^{*}\left(S^{2} \times S^{1} ; \mathbf{Q}\right) \rightarrow$ $H^{*}\left(M^{3} ; \mathbf{Q}\right)$ is injective. Since $H^{1}\left(S^{2} \times S^{1} ; \mathbf{Q}\right) \neq 0$ so also $H^{1}\left(M^{3} ; Z\right) \neq 0$.

Now suppose $H^{1}\left(M^{3} ; Z\right) \neq 0$. Let $v \in H^{1}\left(M^{3} ; Z\right)$ be a primitive element and represent $v$ by a map $f: M^{3} \rightarrow S^{1}$ which is transverse to a basepoint $* \in S^{1}$. Some of the components of $f^{-1}(*)$ must fail to separate $M^{3}$. For if $c$ is a simple closed curve in $M^{3}$, transverse to a submanifold $N^{2}$ which separates $M^{3}$ then one easily argues that $c$ has intersection number 0 with $N^{2}$. Thus if $c$ is chosen so that the Kronecker product $\langle v,\{c\}\rangle=1, c$ must meet some component of $f^{-1}(x)$ in an odd number of points.

Therefore let $N^{2} \subset M^{3}$ be a two-sided nonseparating submanifold and let 
$\hat{M}^{3}$ denote $M^{3}$ cut open along $N^{2}$. So $\partial \hat{M}^{3}$ is the disjoint union $N_{0}^{2} \cup N_{1}^{2}$ of two copies of $N^{2}$. Choose a simple branched covering $\psi: N^{2} \rightarrow S^{2}$. This gives rise to oriented branched coverings $\psi_{i}: N_{i}^{2} \rightarrow S^{2} \times i, i=0$, 1 , where $N_{i}^{2}$ is oriented by its inclusion in $\hat{M}^{3}$ and $S^{2} \times i$ by its inclusion in $S^{2} \times I$.

By the Extension Theorem (6.3), $\psi_{0} \cup \psi$, extends to a simple branched covering $\theta: \hat{M}^{3} \rightarrow S^{2} \times I$. By the Realization Theorem (4.1) the gluing homeomorphism $h: N_{0}^{2} \rightarrow N_{1}^{2}$ by which $M^{3}$ is recovered from $\hat{M}^{3}$ can be realized up to isotopy by one covering a homeomorphism $\bar{h}: S^{2} \times 0 \rightarrow S^{2} \times$ 1 isotopic to the identity. Thus $\theta$ induces the desired branched covering $\varphi$ : $M^{3} \rightarrow S^{2} \times S^{1}$ by gluing the boundary components of $\hat{M}^{3}$ and of $S^{2} \times I$ together using $h$ and $\bar{h}$.

(7.4) REMARK. By (4.5) we may in addition require that the branched coverings $\varphi$ in (7.1) and (7.3) have a connected 1-manifold as branch set, since they are easily seen to be nondegenerate branched coverings.

8. Branched coverings of nonorientable 3-manifolds. In this section we apply the results of $\$ \S 3-6$ to the study of branched coverings of nonorientable 3-manifolds. The end result is that any closed nonorientable 3-manifold is a branched covering of $P^{2} \times S^{1}$ and the possible degrees can be precisely characterized.

Several necessary technical results about the first Stiefel-Whitney class $w_{1}\left(M^{3}\right) \in H^{1}\left(M^{3} ; \mathbf{Z} / 2\right)$ and the representation of its Poincare dual by submanifolds will be proved along the way.

Let $\rho_{2}: H^{*}(X ; \mathbf{Z}) \rightarrow H^{*}(X ; \mathbf{Z} / 2)$ denote reduction $\bmod 2$ and let $\beta$ : $H^{i}(X ; \mathrm{Z} / 2) \rightarrow H^{i+1}(X ; \mathrm{Z})$ denote the Bockstein coboundary homomorphism associated with the coefficient sequence

$$
0 \rightarrow \mathbf{Z} \rightarrow \mathbf{Z} \rightarrow \mathbf{Z} / 2 \rightarrow 0 .
$$

Note that a class $u \in H^{*}(X ; \mathrm{Z} / 2)$ is the reduction of an integral class if and only if $\beta u=0$.

We first consider closed manifolds $M^{3}$ with $w_{1}\left(M^{3}\right) \neq 0$ but $\beta w_{1}\left(M^{3}\right)=0$. The simplest familiar manifold of this sort is the nontrivial $S^{2}$-bundle over $S^{1}$, which we denote by $S^{2} \otimes S^{1}$. Usually we think of $S^{2} \otimes S^{1}$ as $S^{2} \times I$ with $S^{2} \times 0$ and $S^{2} \times 1$ identified by any homeomorphism isotopic to the antipodal map. Sometimes it is also useful to view it as the quotient of $S^{2} \times S^{1}$ by the involution $(x, z) \mapsto(-x,-z)$.

The geometric equivalent of the condition $\beta w_{1}\left(M^{3}\right)=0$ can be expressed in terms of category. A cohomology class $v \in H^{*}(X)$ (any coefficients) has category cat $v \leqslant k$ if $X=X_{0} \cup X_{1} \cup \cdots \cup X_{k}$ where each $X_{i}$ is open (or each $X_{i}$ is closed) and each restriction $v \mid X_{i}=0$.

(8.1) Lemma. Let $v \in H^{1}(X ; \mathbf{Z} / 2)$. Then $\beta v=0$ if and only if cat $v<1$. 
Proof. If $\beta v=0$ then $v$ is the reduction of an integral class and so can be represented by a map $f: X \rightarrow S^{1}$. Then the decomposition $X=f^{-1}\left(S^{1}-\right.$ $\{y\}) \cup f^{-1}\left(S^{1}-\{-y\}\right)$ shows that cat $v \leqslant 1$ since $f \mid f^{-1}\left(S^{1}-\{y\}\right) \cong 0$.

Now suppose cat $v \leqslant 1$. For simplicity we shall assume $X$ is a PL manifold since that is the only case we shall use. Then $X=U_{0} \cup U_{1}$ where $v \mid U_{i}=0$ for $i=0,1$. It is easy to see that we may assume that $U_{0}$ and $U_{1}$ are submanifolds with $U_{0} \cap U_{1}=\partial U_{0}=\partial U_{1}=V$. Then a map $f: X \rightarrow P^{\infty}$ representing $v$ factors homotopically through the suspension $\Sigma V$, say

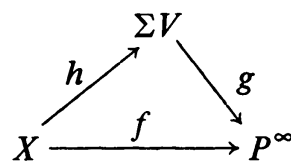

where $h$ collapses the complement of a regular neighborhood of $V$ in $X$ to two points.

The map $\Sigma \Omega P^{\infty} \rightarrow P^{\infty}$, where $\Omega$ is the loop space functor, defined by $[t, \lambda] \mapsto \lambda(t)$, is easily seen to be homotopy equivalent to the standard inclusion $S^{1} \cong P^{1} \subset P^{\infty}$. Then $g: \Sigma V \rightarrow P^{\infty}$ factors through $\Sigma \Omega P^{\infty}$ by $[t, x] \mapsto\left[t, \lambda_{x}\right]$ where $\lambda_{x}(s)=g[s, x]$. Thus $f: X \rightarrow P^{\infty}$ factors homotopically through $P^{1} \subset P^{\infty}$ and so $v$ is integral.

Thus $\beta w_{1}\left(M^{3}\right)=0$ if and only if $M^{3}$ is the union of two orientable submanifolds of codimension 0 .

A submanifold $N^{2} \subset M^{3}$ is said to represent a class $u \in H_{2}\left(M^{3} ; Z / 2\right)$ if $i_{*}\left[N^{2}\right]=u$, where $i: N^{2} \rightarrow M^{3}$ is the inclusion and $\left[N^{2}\right] \in H_{2}\left(N^{2} ; \mathbf{Z} / 2\right)$ is the nonzero element or fundamental class.

(8.2) LEMMA. Let $M^{3}$ be a closed, connected, nonorientable 3-manifold such that $\beta w_{1}\left(M^{3}\right)=0$. Then there is a connected, orientable, two-sided, nonseparating 2-manifold $N^{2} \subset M^{3}$ which represents the Poincare dual $D w_{1}\left(M^{3}\right)$ of $w_{1}\left(M^{3}\right)$ in $H_{2}\left(M^{3} ; \mathbf{Z} / 2\right)$.

Proof. Represent $w_{1}\left(M^{3}\right)$ by a map $f: M^{3} \rightarrow S^{1}$ using the fact that $\beta w_{1}\left(M^{3}\right)=0$. Let $N^{2}=f^{-1}(y)$ where $y$ is a regular value for $f$ (say an interior point of a 1-simplex in a triangulation such that $f$ is simplicial).

Then $N^{2}$ is easily seen to satisfy the statement of the lemma except that $N^{2}$ may not be connected and may separate $M^{3}$. For $N^{2}$ is two-sided, since its normal bundle pulls back from that of $y$ in $S^{1}$, so that $w_{1}\left(N^{2}\right)=w_{1}\left(M^{3}\right) \mid N^{2}$ $=0$, since $f \mid N^{2} \cong 0$; similarly $M^{3}-N^{2}$ is orientable. Therefore generically any orientation-reversing loop in $M^{3}$ must meet $N^{2}$ an odd number of times, so that $N^{2}$ represents $D w_{1}\left(M^{3}\right)$, by the definition of Poincaré duality.

Now $N^{2}$ may be made connected without destroying the above properties by running a minimal number of tubes between components, so that no new 
orientation-reversing loops are added to $N^{2}$.

Finally, assuming as we may that $N^{2}$ is connected, $N^{2}$ cannot separate $M^{3}$. For otherwise any simple closed curve would meet $N^{2}$ in an even number of points.

We can now use (8.2) to construct branched coverings of some nonorientable 3-manifolds.

(8.3) THEOREM. Let $M^{3}$ be a closed, connected, nonorientable 3-manifold such that $\beta w_{1}\left(M^{3}\right)=0$ and let $n \geqslant 3$ be an integer. Then there is a primitive branched covering $\varphi: M^{3} \rightarrow S^{2} \otimes S^{1}$ of degree $n$.

Proof. We view $S^{2} \otimes S^{1}$ as $S^{2} \times I$ with $S^{2} \times 0$ and $S^{2} \times 1$ suitably identified.

Let $N^{2} \subset M^{3}$ be a two-sided, connected, orientable 2-manifold representing $D w_{1}\left(M^{3}\right)$ as guaranteed by (8.2), so that $M^{3}-N^{2}$ is connected and orientable. Let $\hat{M}^{3}$ denote $M^{3}$ cut open along $N^{2}$, so that $\partial \hat{M}^{3}$ is the disjoint union of two copies $N_{0}^{2}$ and $N_{1}^{2}$ of $N^{2}$ and $M^{3}$ is obtained from $\hat{M}^{3}$ by identifying $N_{0}^{2}$ and $N_{1}^{2}$ by a homeomorphism $h: N_{0}^{2} \rightarrow N_{1}^{2}$.

By the Hurwitz Existence Theorem one can construct a simple branched covering $\varphi_{0}: N_{0}^{2} \rightarrow S^{2} \times 0$ of degree $n$. By the Extension Theorem (6.2), $\varphi_{0}$ extends to a branched covering

$$
\hat{\varphi}:\left(\hat{M}^{3} ; N_{0}^{2}, N_{1}^{2}\right) \rightarrow S^{2} \times(I ; 0,1) .
$$

By the Uniqueness Theorem (3.4), $\hat{\varphi} \mid N_{1}^{2}$ is equivalent to $\varphi_{0}$. Therefore by the Realization Theorem (4.1) there are homeomorphisms $f: S^{2} \times 0 \rightarrow S^{2} \times$ 1 and $g: N_{0}^{2} \rightarrow N_{1}^{2}$ such that the diagram

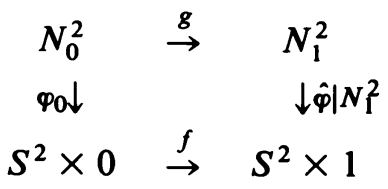

commutes, $g$ is isotopic to $h$, and $f$ is isotopic to the antipodal map. Thus using $g$ we can glue the ends $N_{0}^{2}$ and $N_{1}^{2}$ of $\hat{M}^{3}$ together and obtain the desired branched covering $\varphi: M^{3} \rightarrow S^{2} \otimes S^{1}$, which clearly induces a surjection of fundamental groups.

(8.4) Remark. If $\varphi: M^{3} \rightarrow S^{2} \otimes S^{1}$ is any branched covering then $\beta w_{1}\left(M^{3}\right)=0$. For $\varphi$ preserves first Stiefel-Whitney classes and the decomposition

$$
M^{3}=\varphi^{-1}\left(S^{2} \times\left[0, \frac{1}{2}\right]\right) \cup \varphi^{-1}\left(S^{2} \times\left[\frac{1}{2}, 1\right]\right)
$$

shows that $M^{3}$ is the union of two orientable pieces so that cat $w_{1}\left(M^{3}\right)<1$ and hence $\beta w_{1}\left(M^{3}\right)=0$ by (8.1).

Also one can apply (4.5) to make the branch set of $\varphi$ in (8.3) connected. 
We now turn to the more delicate case when $\beta w_{1}\left(M^{3}\right) \neq 0$. The simplest familiar model of such a manifold is $P^{2} \times S^{1}$.

(8.5) Proposition. Let $M^{3}$ be a connected closed 3-manifold such that $\beta w_{1}\left(M^{3}\right) \neq 0$. Then the Poincare dual $D w_{1}\left(M^{3}\right) \in H_{2}\left(M^{3} ; \mathrm{Z} / 2\right)$ is represented by a connected, orientable, one-sided submanifold $N^{2}$ such that, moreover, the induced homomorphism $H_{1}\left(N^{2} ; Z\right) \rightarrow H_{1}\left(M^{3} ; Z\right)$ is surjective.

Proof. By Thom [T, II.26] (or simply by resolving the singularities in a suitable simplicial 2-cycle) there is a 2-manifold $N^{2} \subset M^{3}$ representing $D w_{1}\left(M^{3}\right)$.

Without changing the mod 2 homology class represented by $N^{2}$ we may add a collection of tubes to $N^{2}$ so that $N^{2}$ becomes connected and $H_{1}\left(N^{2} ; \mathrm{Z}\right)$ $\rightarrow H_{1}\left(M^{3} ; \mathbf{Z}\right)$ is surjective.

Suppose that $N^{2}$ were nonorientable. We shall show that this would imply $\beta w_{1}\left(M^{3}\right)=0$.

Let $W^{3}$ be the total space of the normal disk bundle of $N^{2}$ in $M^{3}$ with $V^{2}=\partial W^{3}$. Then $V^{2}$ is a two-sided submanifold of the orientable manifold $M^{3}-N^{2}$ and hence is orientable. Then the double covering $\pi: V^{2} \rightarrow N^{2}$ induced by the normal bundle projection is necessarily the orientable double covering of $N^{2}$. Therefore, over an annulus in $N^{2}, \pi$ is trivial, while, over a Möbius band in $N^{2}, \pi$ is nontrivial. Thus, over a Möbius band in $N^{2}, W^{3}$ is itself orientable as a 3-manifold. Since any loop in $W^{3}$ may be assumed to lie in $N^{2}$ this shows $W^{3}$ is orientable.

But then $M^{3}=W^{3} \cup\left(M^{3}-N^{2}\right)$ where $W^{3}$ and $M^{3}-N^{2}$ are orientable. By (8.1) this implies $\beta w_{1}\left(M^{3}\right)=0$. Therefore $N^{2}$ must be orientable as desired.

(8.6) Lemma. Let $M^{3}$ be a closed nonorientable 3-manifold. Then $H^{1}\left(M^{3} ; \mathbf{Z}\right)$ $\neq 0$.

Proof. Using $Z / 2$ coefficients it is a standard consequence of Poincaré duality that the mod 2 Euler characteristic $\chi\left(M^{3} ; \mathrm{Z} / 2\right)=0$. But by the Universal Coefficient Theorem the Euler characteristic does not depend on the coefficients used. In particular $\chi\left(M^{3} ; Q\right)=0$. Since $H^{3}\left(M^{3} ; Q\right)=0$ and $H^{0}\left(M^{3} ; \mathbf{Q}\right)=\mathbf{Q}$ it follows that $H^{1}\left(M^{3} ; \mathbf{Q}\right) \neq 0$. Hence $H^{1}\left(M^{3} ; Z\right) \neq 0$.

(8.7) LEMMA. Let $N^{2}$ be a connected, closed, orientable 2-manifold of genus $g>3$, let $f=\left(f_{1}, f_{2}\right): N^{2} \rightarrow P^{1} \times S^{1}$ be a primitive map of degree $n$, and let $k$ be any integer. Then there is a primitive map $f_{1}^{\prime}: N^{2} \rightarrow P^{1}$ such that $f_{1}=f_{1}^{\prime}$ $\bmod 2$ (i.e., if $\cong$ if $f_{1}^{\prime}$ where $i: P^{1} \rightarrow P^{\infty}$ is the inclusion) and $\operatorname{deg}\left(f_{1}^{\prime}, f_{2}\right)=n+$ $2 k$.

Proof. As in the proof of (5.3) we may find a standard basis $\left\{a_{1}, a_{2}, \ldots, a_{g}, b_{1}, b_{2}, \ldots, b_{g}\right\}$ for $H_{1}\left(N^{2} ; Z\right)$ (for which intersection 
numbers satisfy $a_{i} \cdot a_{j}=0=b_{i} \cdot b_{j}$ and $a_{i} \cdot b_{j}=\delta_{i j}$ ) such that $f_{*}$ has a matrix of the form

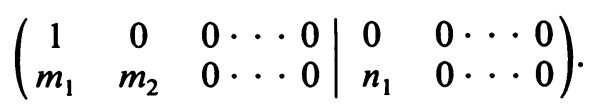

Then $\operatorname{deg}\left(f_{1}, f_{2}\right)=n_{1}=n$.

Since $f_{2^{*}}: H_{1}\left(N^{2} ; Z\right) \rightarrow H_{1}\left(S^{1} ; Z\right)$ is surjective, there are integers $p, q$, and $r$ such that $-p m_{1}-q m_{2}+r n_{1}=1$. Let $f^{\prime}=\left(f_{1}^{\prime}, f_{2}\right)$ be given by the matrix

$$
\left(\begin{array}{ccc|ccc}
1+2 k r & 2 k(p+q+r) & 0 \cdots 0 & 2 k p & 2 k q & 0 \cdots 0 \\
m_{1} & m_{2} & 0 \cdots 0 & n_{1} & 0 & 0 \cdots 0
\end{array}\right) .
$$

Then $\operatorname{deg}\left(f^{\prime}\right)=(1+2 k r) n_{1}-2 k p m_{1}-2 k q m_{2}=n_{1}+2 k$. Clearly $f_{1}^{\prime}$ is primitive.

(8.8) THEOREM. Let $M^{3}$ be a closed, connected 3-manifold such that $\beta w_{1}\left(M^{3}\right)$ $\neq 0$. Then there is a primitive branched covering $M^{3} \rightarrow P^{2} \times S^{1}$ of some degree $n \geqslant 3$.

Proof. By (8.5) there is a connected, orientable, one-sided 2-manifold $N^{2} \subset M^{3}$ representing the Poincare dual $D w_{1}\left(M^{3}\right)$ and such that $H_{1}\left(N^{2} ; \mathbf{Z}\right)$ $\rightarrow H_{1}\left(M^{3} ; \mathrm{Z}\right)$ is surjective. By adding some trivial handles to $N^{2}$ if necessary we may assume that $N^{2}$ has genus $g \geqslant \max \{3, n\}$. Let $W^{3}$ denote the total space of the normal disk bundle $\nu\left(N^{2}\right)$ of $N^{2}$ in $M^{3}$ and let $V^{2}=\partial W^{3}$. The normal bundle $\nu\left(N^{2}\right)$ is classified by a map $N^{2} \rightarrow P^{\infty}$. Since any element of $H^{1}\left(N^{2} ; \mathrm{Z} / 2\right)$ is the reduction of an integral class the classifying map factors through $P^{1} \subset P^{\infty}$ by a map $f_{1}: N^{2} \rightarrow P^{1}$, which is, of course, not uniquely determined.

Let $g_{1}: M^{3} \rightarrow P^{2}$ be the result of applying the Thom-Pontryagin construction to $f_{1}$, so that $g_{1}$ maps $W^{3}$ by a bundle map to the Möbius band $\Re^{2}$ which is the total space of the normal bundle of $P^{1}$ in $P^{2}$ and maps $M^{3}-W^{3}$ into $D^{2}=P^{2}-\mathfrak{N}^{2}$. Then $g_{1}$ represents $w_{1}\left(M^{3}\right)$.

Let $g_{2}: M^{3} \rightarrow S^{1}$ represent a primitive cohomology class $v \in H^{1}\left(M^{3} ; Z\right)$, which exists by (8.6).

Let $f_{2}=g_{2} \mid N^{2}$. Then we have a commutative diagram

$$
\begin{array}{ccc}
M^{3} & \stackrel{g=\left(g_{1}, g_{2}\right)}{\rightarrow} & P^{2} \times S^{1} \\
\cup & & \cup \\
N^{2} & f=\left(f_{1}, f_{2}\right) & P^{1} \times S^{1}
\end{array}
$$

and $g \mid W^{3}: W^{3} \rightarrow \Re^{2} \times S^{1}$ is a bundle map.

Note that $\operatorname{deg}(f)=\operatorname{deg}(g)$. For let $\tilde{M}^{3}$ denote the orientable double covering of $M^{3}$ and let $\tilde{W}^{3}, \tilde{V}^{2}$, and $\tilde{N}^{2}$ denote the induced (nontrivial) double coverings of $W^{3}, V^{2}$, and $N^{2}$. Also let $\tilde{g}: \tilde{M}^{3} \rightarrow S^{2} \times S^{1}$ be a lift of $g$ 
and $\tilde{f}: \tilde{N}^{2} \rightarrow S^{1} \times S^{1}$ be $\tilde{g} \mid \tilde{N}^{2}$. Then by definition $\operatorname{deg}(g)=\operatorname{deg}(\tilde{g})$ and by excision $\operatorname{deg}(\tilde{g})=\operatorname{deg}\left(\tilde{g} \mid\left(\tilde{W}^{3}, \tilde{V}^{2}\right)\right)$. But $\tilde{N}^{2}$ has a trivial normal bundle in $\tilde{W}^{3}$ so clearly

$$
\operatorname{deg}\left(\tilde{g} \mid\left(\tilde{W}^{3}, \tilde{V}^{2}\right)\right)=\operatorname{deg}(\tilde{f}) .
$$

Also $2 \operatorname{deg}(\tilde{f})=2 \operatorname{deg}(f)$, so that $\operatorname{deg}(g)=\operatorname{deg}(f)$, as desired.

The map $g$ is easily seen to be primitive, since $w_{1}\left(M^{3}\right)$ is not an integral class. The map $f$ is primitive since $f_{1}$ is clearly primitive and $f_{2}$ is primitive because $g_{2}$ and $H_{1}\left(N^{2} ; Z\right) \rightarrow H_{1}\left(M^{3} ; \mathrm{Z}\right)$ is surjective.

By (8.7) we can alter $f_{1}$ (and hence $g_{1}$ ) to obtain an integer $n \geqslant 3$ (say 3 or 4) for $\operatorname{deg}(f)=\operatorname{deg}(g)$. For the degree of $f$ can be altered by any even integer and still have $f_{1}$ classify the normal bundle $\nu\left(N^{2}\right)$ and $f$ be primitive.

Now by (5.1) $f$ is homotopic to a simple local branched covering of degree $n$. Since $f=\left(f_{1}, f_{2}\right)$ is only determined up to homotopy we may assume $f$ is a simple local branched covering.

Since $g \mid W^{3}: W^{3} \rightarrow \Re^{2} \times S^{1}$ is a bundle map it too is a simple branched covering, as is $g \mid V^{2}: V^{2} \rightarrow S^{1} \times S^{1}$. Now $g \mid V^{2}$ is clearly at least a semi-local branched covering. Therefore, by (6.5) $g \mid V^{2}$ extends to a simple branched covering $M^{3}-$ int $W^{3} \rightarrow D^{2} \times S^{1}$ where $D^{2} \times S^{1}=P^{2} \times S^{1}-$ int $\mathfrak{N}^{2} \times$ $S^{1}$, since $g \mid V^{2}$ extends as a map. This extension, together with $g \mid W^{3}$, defines the desired branched covering $M^{3} \rightarrow P^{2} \times S^{1}$.

(8.9) REMARK. The branched coverings in (8.8) can be altered, using (4.5), so as to have connected branch set.

(8.10) Corollary. Any closed nonorientable 3-manifold $M^{3}$ is a branched covering of $P^{2} \times S^{1}$ of degree $\leqslant 6$.

PRoof. If $\beta w_{1}\left(M^{3}\right) \neq 0$ this follows from (8.8). If $\beta w_{1}\left(M^{3}\right)=0$ there is a branched covering $\varphi: M^{3} \rightarrow S^{2} \otimes S^{1}$, and the result follows by composing $\varphi$ with the double covering $S^{2} \otimes S^{1} \rightarrow P^{2} \times S^{1}$.

(8.11) REMARK. It is somewhat awkward to describe the degrees which can be achieved by primitive branched coverings $M^{3} \rightarrow P^{2} \times S^{1}$. The simplest way to put it is that a given degree $n \geqslant 3$ can be achieved if and only if there is a primitive map $f: M^{3} \rightarrow P^{2} \times S^{1}$ such that $\operatorname{deg} f=n$ and $f^{*} w_{1}\left(P^{2} \times S^{1}\right)$ $=w_{1}\left(M^{3}\right)$. Any such map $M^{3} \rightarrow P^{2} \times S^{1}$ is determined $\bmod 2$ by $w_{1}\left(M^{3}\right)$ in the first factor and by an element of $H^{1}\left(M^{3} ; Z\right)$ in the second. One can without difficulty show that in the proof of $(8.8)$ one can achieve the following results:

(i) If $\beta w_{1}\left(M^{3}\right) \neq 0$, then there is a primitive branched covering $M^{3} \rightarrow P^{2}$ $\times S^{1}$ of any odd degree greater than or equal to 3 if and only if there is an element $v \in H^{1}\left(M^{3} ; Z\right)$ such that $\rho_{2}(v) w_{1}\left(M^{3}\right)^{2} \neq 0$.

(ii) If $\beta w_{1}\left(M^{3}\right) \neq 0$, then there is a primitive branched covering $M^{3} \rightarrow P^{2}$ 
$\times S^{1}$ of any even degree greater than 3 if and only if there is a primitive $v \in H^{1}\left(M^{3} ; Z\right)$ such that $\rho_{2}(v) w_{1}\left(M^{3}\right)^{2}=0$ (e.g. rank $\left.H^{1}\left(M^{3} ; Z\right)>1\right)$.

One can also carry through the proof of (8.8) in some cases when $\beta w_{1}\left(M^{3}\right)$ $=0$.

(iii) If $w_{1}\left(M^{3}\right) \neq 0$ and $\beta w_{1}\left(M^{3}\right)=0$, then there is a primitive branched covering $M^{3} \rightarrow P^{2} \times S^{1}$ of any even degree greater than 3 if and only if rank $H^{1}\left(M^{3} ; Z\right)>1$.

Thus the only nonorientable 3-manifolds $M^{3}$ which are not primitive branched coverings of $P^{2} \times S^{1}$ are those with $\beta w_{1}\left(M^{3}\right)=0$ and $H^{1}\left(M^{3} ; Z\right)$ $\approx \mathbf{Z}$.

(8.12) ReMARK. A refinement of the class of nonorientable 3-manifolds emerges from the preceding discussion:

I. $w_{1}\left(M^{3}\right) \neq 0$ but $\beta w_{1}\left(M^{3}\right)=0$;

II. $\beta w_{1}\left(M^{3}\right) \neq 0$ but $w_{1}\left(M^{3}\right)^{2}=0$;

III. $w_{1}\left(M^{3}\right)^{2} \neq 0$ but $\rho_{2}(v) w_{1}\left(M^{3}\right)^{2}=0$ for all $v \in H^{1}\left(M^{3} ; \mathbf{Z}\right)$;

IV. $\rho_{2}(v) w_{1}\left(M^{3}\right)^{2} \neq 0$ for some $v \in H^{1}\left(M^{3} ; \mathbf{Z}\right)$.

These subclasses are all nonempty. One can also interpose an infinite sequence of subclasses in I by considering Bockstein homomorphisms associated with the coefficient sequence $0 \rightarrow \mathbf{Z} / 2^{k} \rightarrow \mathbf{Z} / 2^{k+1} \rightarrow \mathbf{Z} / 2 \rightarrow 0$, for $k \geqslant 2$.

9. Open book decompositions. In this section we shall apply the results of $\S 8$ to prove the existence of open book decompositions for nonorientable 3manifolds.

Recall that an open book decomposition for $M^{3}$ consists of a 1-manifold $\mathscr{B}^{1} \subset M^{3}$, called the binding, with trivialized normal bundle $\mathscr{B}^{1} \times D^{2}$ and a fibering of $\mathcal{E}=M^{3}-\mathscr{B}^{1}, p: \mathcal{E} \rightarrow S^{1}$, such that $\left.p\right|^{1} \times\left(D^{2}-0\right)$ has the form $(x, z) \mapsto z /\|z\|$. The fibers $F_{z}=p^{-1}(z)$ are called the pages or leaves. Then the closure $\bar{F}_{z}$ is a submanifold with $\partial F_{z}=\mathscr{B}^{1}$. It will sometimes be more convenient to work with $E=M^{3}-\operatorname{int}\left(\mathscr{B}^{1} \times D^{2}\right) ; \mathcal{E}$ is homeomorphic to $E$ with an open collar attached.

In [A2] Alexander used his theorem that every closed orientable 3-manifold $M^{3}$ is a branched covering of $S^{3}$ to show that $M^{3}$ admits an open book decomposition. In similar fashion we shall use (8.10) to prove the analogous statement for nonorientable 3-manifolds. In outline the argument is as follows: First we show that $P^{2} \times S^{1}$ admits an open book decomposition; second we show that the branch set of a simple branched covering over a 3-manifold with an open book decomposition can be adjusted so as to be transverse to the leaves; and, finally, we show that an open book decomposition can be pulled back through such a transverse branched covering.

(9.1) THEOREM. The manifold $P^{2} \times S^{1}$ admits an open book decomposition. 
Proof. We shall use Stallings' Fibering Theorem [Sta] to deduce the existence of the decomposition rather than explicitly exhibiting it.

Let $\mathscr{B}^{1}$ be a circle in $P^{2} \times S^{1}$ which winds twice about the $S^{1}$ factor. Precisely, we may view $P^{2} \times S^{1}$ as $P^{2} \times I$ with $P^{2} \times 0$ identified with $P^{2} \times 1$ using any homeomorphism $f: P^{2} \times 0 \rightarrow P^{2} \times 1$ which is isotopic to the identity. Let $D^{2} \subset P^{2}$ be a 2-disk, let $x_{1}, x_{2} \in$ int $D^{2}$, and let $D_{1}$ and $D_{2}$ be disjoint disk neighborhoods of $x_{1}$ and $x_{2}$ in int $D^{2}$. Now let $f: P^{2} \rightarrow P^{2}$ be a homeomorphism which is the identity outside $D^{2}$ and permutes $\left(D_{1}, x_{1}\right)$ and $\left(D_{2}, x_{2}\right)$. Then $\mathscr{B}^{1}$ is the image of $x_{1} \times I \cup x_{2} \times I$ in $P^{2} \times I / f$ and $\mathscr{B}^{1} \times D^{2}$ is the image of $D_{1} \times I \cup D_{2} \times I$. Set $\mathcal{E}^{2}=P^{2} \times S^{1}-\mathscr{B}^{1}$ and $E=P^{2} \times S^{1}-\operatorname{int}\left(\Re^{1} \times D^{2}\right)$.

According to [Sta] it suffices to show that $E$ is irreducible and that the surjective homomorphism $\pi_{1}(\partial E) \rightarrow Z$ induced by the projection $\mathscr{B}^{1} \times S^{1} \rightarrow$ $S^{1}$ extends to a homomorphism $\pi_{1}(E) \rightarrow \mathbf{Z}$ with finitely generated kernel not equal to $\mathbf{Z} / 2$.

To see that $E$ is irreducible note that $E$ is covered by the product of a triply punctured 2-disk with $\mathbf{R}$, which is easily seen to be irreducible.

Let $N^{2}=P^{2}-\operatorname{int}\left(D_{1} \cup D_{2}\right)$ and let $* \in \partial D^{2} \subset N^{2}$ be a base point. Then $\pi_{1}\left(N^{2}, *\right)$ has a presentation $\left\langle a, b, c: a b=c^{2}\right\rangle$, where $a$ and $b$ correspond to the loops $\partial D_{1}$ and $\partial D_{2}$ suitably connected to $*$ and $c$ represents a generator of $\pi_{1}\left(P^{2}, *\right)$ lying outside $D^{2}$. Then (with proper choice of labeling) $f_{*}: \pi_{1}\left(N^{2}, *\right)$ $\rightarrow \pi_{1}\left(N^{2}, *\right)$ is given by $a \mapsto a b a^{-1}, b \mapsto a$, and $c \mapsto c$.

By Van Kampen's Theorem then $\pi_{1} E$ has a presentation

$$
\left\langle a, b, c, g: a b=c^{2}, g a g^{-1}=a b a^{-1}, g b g^{-1}=a, g c g^{-1}=c\right\rangle .
$$

Also $\pi_{1}(\partial E, *)$ is given by $\left\langle x, y: y x y^{-1}=x\right\rangle$ and the inclusion-induced homomorphism $\pi_{1}(\partial E, *) \rightarrow \pi_{1}(E, *)$ is given by $x \mapsto a$ and $y \mapsto g^{2}$ (with suitable choices made), while the projection $\pi_{1}(\partial E, *) \rightarrow \mathbf{Z}$ is given by $x \mapsto 1$ and $y \mapsto 0$.

Define $\alpha: \pi_{1}(E, *) \rightarrow \mathbf{Z}$ by

$$
a \mapsto 1, \quad b \mapsto 1, \quad c \mapsto 1, \quad g \mapsto 0 .
$$

It is immediate that $\alpha$ is well-defined and extends the projection $\pi_{1}(\partial E, *)$ $\rightarrow \mathbf{Z}$. We claim $\operatorname{ker}(\alpha)$ is the free group on two generators. To prove the claim we modify the presentation of $\pi_{1}(E, *)$. First, since $b=a^{-1} c^{2}$, we can write

$$
\pi_{1}(E, *)=\left\langle a, c, g: g a g^{-1}=c^{2} a^{-1}, g c g^{-1}=c\right\rangle .
$$

Now make the following substitutions: $u=g, v=a c^{-1}, w=c g^{-1}$. Then $\pi_{1}(E, *)=\left\langle u, v, w: v w=w v^{-1}, u w=w v\right\rangle$ and $\alpha: \pi_{1}(E, *) \rightarrow \mathbf{Z}$ is given by $u \mapsto 0, v \mapsto 0, w \mapsto 1$. The relations then show that any word $r$ in $u, v$, and $w$ can be put in the form $r=r_{0} w^{n}$ where $\alpha(r)=n$ and $r_{0}$ is a word in $u$ and $v$. Thus 
$\operatorname{ker}(\alpha)$ is generated by $u$ and $v$ and hence is the free group on two generators.

(9.2) Remark. The typical leaf $F_{z}$ of the so-determined open book decomposition for $P^{2} \times S^{1}$ must be nonorientable since $\mathscr{B}^{1}=\partial \bar{F}_{z}$ and $\mathscr{B}^{1}$ clearly does not bound integrally. Thus one deduces that $F_{z}$ is the punctured Klein bottle and that the monodromy map $g: F_{z} \rightarrow F_{z}$ for the bundle $\mathcal{E}$ is the " $Y$-homeomorphism" of Lickorish [Li1] (since the other possible choices for $g$ do not yield the right fundamental group).

(9.3) THEOREM. Let $M^{3}$ be a closed 3-manifold with open book decomposition $\mathscr{B}^{1} \times D^{2} \cup \mathcal{E}, F^{2} \hookrightarrow \mathcal{E} \stackrel{p}{\rightarrow} S^{1}$, and let $L \subset M^{3}$ be a tame link. Then there is an ambient isotopy $H_{t}: M^{3} \rightarrow M^{3}$ such that $H_{0}$ is the identity, $H_{1}(L) \subset \mathcal{E}$, and $p \mid H_{1}(L): H_{1}(L) \rightarrow S^{1}$ is a covering map (i.e., $H_{1}(L)$ meets the leaves of $\mathcal{E}$ transversely).

Proof. Our proof generalizes that of Alexander [A2] for the case where $M^{3}=S^{3}$ with the standard open book decomposition $S^{1} \times D^{2} \cup \mathbf{R}^{2} \times S^{1}$, but is rather more complicated.

By a simple general position argument we may assume to begin with that $L \subset \mathcal{E}$ and that the restriction $p \mid L: L \rightarrow S^{1}$ embeds each simplex in some triangulation and so fails to be a covering map only at finitely many points which we shall refer to as fold points. The idea is to eliminate these fold points in pairs by pulling $L$ across $\mathscr{B}^{1}$.

Orient a component of $L$, let $A$ and $B$ be points in that component, and let $\sigma$ be the arc in $L$ from $A$ to $B$. Assume int $\sigma$ contains no fold points of $L$. Suppose there is a 2-simplex $\Delta$ in $M^{3}$ with one edge $\sigma$, and opposite vertex $v \in \mathscr{B}^{1}$ and which meets a leaf in $M^{3}$, if at all, in a single arc which spans from $\sigma$ to $v$. Call such a 2-simplex $\Delta$ a spanning disk. (Call $\Delta$ a clean spanning disk if it should happen in addition that $\Delta \cap L=\sigma$.)

We shall prove the following three statements:

(9.4) There is an isotopy (locally unknotted) $f_{t}: L \rightarrow M^{3}$ such that $f_{t}$ is fixed on $\sigma$ and supported on a neighborhood of $L \cap$ int $\Delta$ and such that $f_{1}(L) \cap \Delta$ $=\sigma, f_{1}(L) \subset E, p \mid f_{1}(L)$ is finite-to-one and $f_{1}(L)$ has exactly the same fold points as $L$. (This makes $\Delta$ clean.)

(9.5) If $A$ and $B$ are fold points and $\Delta$ is a clean spanning disk then there is a locally unknotted isotopy $g_{t}: L \rightarrow M^{3}$ such that $g_{t}$ is supported on $\sigma, g_{1}(L)$ has two fewer fold points than $L$, and $p \mid g_{1}(L)$ is still finite-to-one. (This eliminates a pair of adjacent folds).

(9.6) If $B$ is the only fold point on $\sigma$ and $\Delta$ is a clean spanning disk then there is a locally unknotted isotopy $h_{t}: L \rightarrow M^{3}$ such that $h_{t}$ is supported on $\sigma$ 
and such that $h_{1}(L)$ has exactly the same fold points as $L$ except that the fold at $B$ now occurs at $A$. (This pushes a fold point along $L$ until it is closer to another fold.)

Given (9.4)-(9.6) the proof is completed as follows. Clearly each component of $L$ contains an even number of fold points. By induction it suffices to eliminate a successive pair $C, D$ of fold points. The interval $[C, D]$ in $L$ may not lie on a clean spanning disk. However, there does exist a partition $C=x_{0}<x_{1}<\cdots<x_{n}=D$ of $[C, D]$ such that each $\left[x_{i}, x_{i+1}\right]$ lies on a clean spanning disk $\Delta_{i}$. This follows from the compactness of $[C, D]$ since each point $x \in L$ lies in an interval in $L$ which lies in a clean spanning disk obtained by splitting a tubular neighborhood of an arc from $x$ to a point of $\mathscr{B}^{1}$ which misses the rest of $L$ and $\mathscr{B}^{1}$ and lies in a single leaf.

Then by (9.6) the fold at $D=x_{n}$ can be moved to $x_{n-1}$ using $\Delta_{n-1}$ without introducing new folds. Unfortunately the new $\left[x_{n-1}, x_{n}\right]$ may intersect $\Delta_{n-2}$. By (9.4) $\left[x_{n-1}, x_{n}\right]$ can be moved off $\Delta_{n-2}$. Continuing in this way the fold at $x_{n-1}$ is moved to $x_{n-2}$, etc., until it has been moved to $x_{2}$. Then the pair of folds at $x_{1}$ and $x_{0}$ can be eliminated by (9.5) after applying (9.4) to move the new $\left[x_{1}, x_{n}\right]$ off of $\Delta_{0}$. An application of the isotopy extension theorem completes the proof.

PROOF OF (9.4). By general position we may assume that $L-\sigma$ meets $\Delta$ transversely in finitely many nonfold points in int $\Delta$. Folds or points of tangency may be moved off $\Delta$ slightly in a bicollar $\Delta \times[-1,1]$ around $\Delta$ and an $\operatorname{arc}$ of $L-\sigma$ lying in $\Delta$ may be tilted in $\Delta \times[-1,1]$ so as to meet $\Delta$ in an isolated point of transverse intersection. In addition we continue to keep $L \subset \mathcal{E}$.

Now consider an innermost point $z \in(L-\sigma) \cap \Delta$ in the sense that the line segment from $v$ to $z$ in $\Delta$ which runs along a single leaf of $M^{3}$ meets no other point of $L$. Let $\tau$ be a very small arc in $L$ which lies in $\Delta \times[-1,1]$ such that $z \in$ int $\tau$ and $\tau$ contains no fold points. Replace $\tau$ by an arc $\tau^{\prime}$ which spins once around $\mathfrak{B}^{1}$ near $v$ instead of cutting through $\Delta$. This can be accomplished by an isotopy supported on $\tau$ simply by sliding $z$ in $\Delta$ to $v$ and then slightly off $\Delta$.

Continuing in this way we eventually eliminate all points of $(L-\sigma) \cap \Delta$.

ProOF OF (9.5) AND (9.6). Shrinking in along leaves and reparametrizing $\mathscr{B}^{1} \times D^{2}$ we may assume $\Delta$ is a sector of $v \times D^{2}$ and $L \cap\left(\mathscr{B}^{1} \times D^{2}\right)=\sigma$. Let $g_{t}$ be the isotopy supported on $\sigma$ which moves $\sigma$ to the complementary arc $\sigma^{\prime}=\left(v \times \partial D^{2}\right)-$ int $\sigma$. If $A$ and $B$ are fold points this eliminates the two folds, proving (9.5). If $B$ is a fold and $A$ is not, this moves the fold at $B$ to $A$, proving (9.6).

This completes the proof of (9.3). 
The following is a precise statement of an observation in [A2].

(9.7) Proposition. Let $\varphi: M^{3} \rightarrow N^{3}$ be a branched covering and let $\mathscr{B}^{1} \times$ $D^{2} \cup \mathcal{E}, p: \mathcal{E} \rightarrow S^{1}$, be an open book decomposition for $N^{3}$. Suppose the branch set of $\varphi$ is a 1-manifold $L \subset \mathcal{E} \subset N^{2}$ transverse to the leaves of $N^{3}$. Then

$$
M^{3}=\varphi^{-1}\left(\mathscr{B}^{1}\right) \times D^{2} \cup \varphi^{-1}(\mathcal{E}), \quad p \varphi: \varphi^{-1}(\mathcal{E}) \rightarrow S^{1},
$$

defines an open book decomposition of $M^{3}$.

Proof. Covering space theory implies that $\varphi^{-1}\left(\mathscr{B}^{1}\right)$ is a 1 -manifold with trivial normal bundle pulled back from that of $\mathscr{B}^{1}$ in $N^{3}$. To see that $p \varphi$ : $\varphi^{-1}(\mathcal{E}) \rightarrow S^{1}$ is a bundle, we just note that any leaf $F_{z}$ of $\mathcal{E}$ in $N^{3}$ has a neighborhood $U$ such that the pair $(U, L \cap U) \cong\left(F_{z} \times \mathbf{R},\left(L \cap F_{z}\right) \times \mathbf{R}\right)$ by the transversality condition. Therefore over such a $U, \varphi$ also splits as a product with $\mathbf{R}$. The proposition follows easily from this.

(9.8) ThEOREM. Any closed 3-manifold $M^{3}$ admits an open book decomposition.

Proof. If $M^{3}$ is orientable this is due to Alexander. So assume $M^{3}$ is nonorientable. By (8.9) and (6.5) there is a simple branched covering $\varphi$ : $M^{3} \rightarrow P^{2} \times S^{1}$ with branch set $L$ a 1 -manifold, since $\varphi$ is simple. By (9.1) $P^{2} \times S^{1}$ admits an open book decomposition. By (9.3) we may assume, after composing $\varphi$ with an isotopy of $N^{3}$, that $L$ meets the leaves transversely. Therefore by (9.7) $\varphi$ induces on $M^{3}$ the desired open book decomposition.

(9.9) Remarks. (i) By spinning $L$ more times around $\mathscr{B}^{1} \cong S^{1}$ in $P^{2} \times S^{1}$ as in the proof of (9.3) we may make the induced binding $\varphi^{-1}\left(\Re^{1}\right)$ connected at the expense of raising the genus of the leaves. Alternatively this observation follows from a general remark of González-Acuña.

(ii) Another approach to (9.8) would be one similar to that used by J. Wood [Wo] to construct codimension one foliations on nonorientable 3-manifolds.

10. Knots as branched coverings of the trivial knot. Let $K \subset S^{3}$ be a knot and let $C \subset S^{3}$ be a trivial knot.

(10.1) THEOREM. For any integer $n \geqslant 3$ there exists a simple branched covering $\varphi: S^{3} \rightarrow S^{3}$ of degree $n$ such that $K=\varphi^{-1}(C)$ and the branch set of $\varphi$ is a knot in $S^{3}-C$.

Proof. Let $K \times D^{2}$ and $C \times D^{2}$ denote trivialized normal bundles for $K$ and $C$ in $S^{3}$. Then $S^{3} \cong C \times D^{2} \cup D^{2} \times S^{1}$. Let $V^{2} \subset S^{3}-K \times$ int $D^{2}$ be a Seifert surface for $K$ such that

$$
\partial V^{2}=K \times *, \quad * \in \partial D^{2} .
$$


Let $K \rightarrow C$ be an $n$-fold covering and extend it to $K \times D^{2} \rightarrow C \times D^{2}$ by the identity map on $D^{2}$. Then we have an $n$-fold covering $\partial V^{2} \rightarrow \partial D^{2} \times *$. By the 2-dimensional extension theorem (6.4) this extends to a simple branched covering $V^{2} \rightarrow D^{2} \times *$ of degree $n$.

Let $W^{3}$ denote $S^{3}-\left(K \times\right.$ int $\left.D^{2}\right)$ cut open along $V^{2}$. Note that $D^{2} \times S^{1}$ cut open along $D^{2} \times *$ is homeomorphic to $D^{2} \times I$. Thus we obtain a branched covering $\partial W^{3} \rightarrow \partial\left(D^{2} \times I\right)$. By (6.3) this extends to a branched covering $W^{3} \rightarrow D^{2} \times I$. Gluing the pieces back together, we obtain the desired branched covering $S^{3} \rightarrow S^{3}$. (If the branch set is not connected, it can be made connected by (4.5).)

(10.2) RemarK. D. Goldsmith asked whether there is a map $f:\left(S^{3}, K\right) \rightarrow$ $\left(S^{3}, C\right)$ such that $f^{-1}(C)=K$ and $f$ is a covering off of a 1-complex, and (10.1) answers this question affirmatively.

(10.3) REMARK. A similar argument shows that if $L \subset S^{3}$ is a tame link of $k$ components, then for any $n \geqslant \max \{3, k\}$ there is a branched covering $\varphi$ : $S^{3} \rightarrow S^{3}$ of degree $n$, such that $L=\varphi^{-1}(C)$ and $B_{\varphi}$ is a knot in $S^{3}-C$.

Appendix. Branched coverings of nonorientable manifolds in higher dimensions. Here we show that a nonorientable $2 n$-dimensional PL manifold can be expressed as a branched covering of real projective space $P^{2 n}$. This result reportedly appears in unpublished work of $R$. H. Fox. When $n=1$ it is due to Wille [Wi]. We also show that there can be no such universal target in odd dimensions greater than 3 .

The result is formulated in terms of equivariant branched coverings between manifolds with a free involution and incorporates techniques of Alexander [A1] and of Heins [He].

(A.1) ThEOREM. Let $M^{n}$ be an orientable PL $n$-manifold without boundary and let $T: M^{n} \rightarrow M^{n}$ be a fixed point free $P L$ involution such that $T$ preserves orientation if $n$ is odd and reverses orientation if $n$ is even. Then there is a $\mathrm{Z}_{2}$-equivariant branched covering $\left(M^{n}, T\right) \rightarrow\left(S^{n}, A\right)$ where $A$ is the antipodal involution of $S^{n}$.

(A.2) CoRollary. Let $N^{2 n}$ be a nonorientable PL 2n-manifold without boundary. Then there is a branched covering $N^{2 n} \rightarrow P^{2 n}$.

Proof of (A.2). Apply (1) to the orientable double cover $M^{2 n} \rightarrow N^{2 n}$ with deck transformation $T$ to obtain an equivariant branched covering $\left(M^{2 n}, T\right)$ $\rightarrow\left(S^{2 n}, A\right)$ and hence, upon passage to orbit spaces, a branched covering $N^{2 n} \rightarrow P^{2 n}$.

(A.3) Complement. If $n$ is odd and greater than 3 there is no single $n$-manifold $N^{n}$ such that every nonorientable $n$-manifold is a branched covering over $N^{n}$. 
Proof of (A.3). Throughout homology will be taken with rational coefficients. Let $N^{n}$ be any nonorientable closed $n$-manifold and $\tilde{N}^{n} \rightarrow N^{n}$ be its orientable double covering, with deck transformation $T: \tilde{N}^{n} \rightarrow \tilde{N}^{n}$. Since $n$ is odd, a standard Poincaré duality argument and the universal coefficient theorem show as in (8.6) that the Euler characteristic $\chi\left(N^{n}\right)=0$. Therefore $H_{j}\left(N^{n}\right) \neq 0$ for some odd $j<n$, since $H_{0}\left(N^{n}\right)=\mathbf{Q}$ while $\mathbf{H}_{n}\left(N^{n}\right)=0$.

Now $H_{*}\left(N^{n}\right) \approx H_{*}\left(\tilde{N}^{n}\right)^{T *}$, the $T_{*}$-invariant homology of $\tilde{N}^{n}$ [B, p. 38]. Therefore $H_{j}\left(\tilde{N}^{n}\right) \neq 0$ also. Then Poincaré duality implies that $H_{2 i}\left(\tilde{N}^{n}\right) \neq 0$ for some $i>0$.

Choose $2 k=2 i \pm 2$ so that $0<k<n$, using $n \geqslant 5$. Then $P^{2 k} \times S^{n-2 k}$ cannot be expressed as a branched covering of $N^{n}$. For then $S^{2 k} \times S^{n-2 k}$ would be a branched covering of $\tilde{N}^{n}$. Now any branched covering between closed orientable manifolds has finite degree and so induces a surjection on rational homology by a well-known argument using Poincaré duality. But $H_{2 i}\left(S^{2 k} \times S^{n-2 k}\right)=0$ while $H_{2 i}(\tilde{N}) \neq 0$, completing the proof.

Proof of (A.1). Triangulate $M$ so that $T$ is simplicial and arbitrarily partition and index the $k$-simplices of $M, 0 \leqslant k \leqslant n$, to form two sets $\left\{\sigma_{i}^{k}\right\}$ and $\left\{\tau_{i}^{k}\right\}$ such that $T \sigma_{i}^{k}=\tau_{i}^{k}$ (and so $T \tau_{i}^{k}=\sigma_{i}^{k}$ ).

Let $M^{\prime}$ denote the first barycentric subdivision of $M$. A typical $k$-simplex of $M^{\prime}$ then has the form $\hat{\rho}_{0} \hat{\rho}_{1} \cdots \hat{\rho}_{k}$ where $\rho_{0}<\rho_{1}<\cdots<\rho_{k}$ are simplices of $M$ and $\hat{\rho}_{i}$ denotes the barycenter of $\rho_{i}$. Then $T$ is still simplicial on $M^{\prime}$ and the above partition induces a partition of the vertices of $M^{\prime}$ into $2(n+1)$ sets.

Let $e_{k}=(0, \ldots, 0,1,0, \ldots, 0)$ denote the standard $k$ th basis vector in $\mathbf{R}^{n+1}$ and view $S^{n}$ as the boundary of the convex hull of $\left\{ \pm e_{k}: 1 \leqslant k \leqslant n+\right.$ 1). This triangulates $S^{n}$ as the join of $(n+1)$ copies $\pm e_{k}$ of $S^{0}$. The antipodal map $A$ is realized as the linear extension of $\pm e_{k} \rightarrow \mp e_{k}$ on vertices.

Begin to define $\varphi: M^{\prime} \rightarrow S^{n}$ on vertices by mapping $\hat{\sigma}_{i}^{k} \mapsto e_{k}$ and $\hat{\tau}_{i}^{k} \mapsto-e_{k}$. Extend this map over the $(n-1)$-skeleton of $M^{\prime}$ by linearity on simplices, noting that no pair $e_{k}$ and $-e_{k}$ appears as the image of a pair of vertices of a single simplex of $M^{\prime}$, since they are both images of barycenters of $k$-simplices of $M$.

To extend this map $\varphi$ over the $n$-simplices, orient $M$ and $S^{n}$, thus orienting the $n$-simplices of each. Now consider an $n$-simplex $\hat{\rho}_{0} \cdots \hat{\rho}_{n}$ of $M^{\prime}$ and its $T$-image $T \hat{\rho}_{0} \ldots T \hat{\rho}_{n}$.

If the natural linear extension of $\varphi$ over $\hat{\rho}_{0} \cdots \hat{\rho}_{n}$ is orientation-preserving, then so is the linear extension of $\varphi$ over $T \hat{\rho}_{0} \cdots T \hat{\rho}_{n}$ since $T$ and $A$ are either both orientation-preserving, or both orientation-reversing. In this case extend $\varphi$ linearly over both $\hat{\rho}_{0} \cdots \hat{\rho}_{n}$ and $T \hat{\rho}_{0} \cdots T \hat{\rho}_{n}$.

If the natural linear extension of $\varphi$ over $\hat{\rho}_{0} \cdots \hat{\rho}_{n}$ is orientation-reversing 
so is that over $T \hat{\rho}_{0} \ldots T \hat{\rho}_{n}$. Note that both $\overline{S^{n}-\varphi \hat{\rho}_{0} \cdots \varphi \hat{\rho}_{n}}$ and $\overline{S^{n}-\varphi T \hat{\rho}_{0} \cdots \varphi T \hat{\rho}_{n}}=\overline{S^{n}-A \varphi \hat{\rho}_{0} \cdots A \varphi \hat{\rho}_{n}}$ are PL $n$-cells. In this case extend $\varphi: \partial\left(\hat{\rho}_{0} \cdots \hat{\rho}_{n}\right) \rightarrow \partial\left(\varphi \hat{\rho}_{0} \cdots \varphi \hat{\rho}_{n}\right)$ to a PL homeomorphism $\hat{\rho}_{0} \cdots \hat{\rho}_{n} \rightarrow \overline{S^{n}-\varphi \hat{\rho}_{0} \cdots \varphi \hat{\rho}_{n}}$, and then, having made this extension, extend $\varphi$ over $T \hat{\rho}_{0} \cdots T \hat{\rho}_{n}$ by $A \varphi T$.

The resulting $\varphi: M \rightarrow S^{n}$ is clearly a well-defined, $\mathbf{Z}_{2}$-equivariant, nondegenerate, PL map. To see that $\varphi$ is open it suffices to check that there are no folds: Any open $(n-1)$-simplex of $M^{\prime}$ has a neighborhood in $M$ which $\varphi$ embeds in $S^{n}$. This follows immediately from the definition of $\varphi$ and completes the proof.

One might make the conjecture that a given nonorientable $(2 n+1)$ manifold is a branched covering of a twisted product of spheres and/or projective spaces.

\section{REFERENCES}

[A1] J. W. Alexander, Note on Riemann spaces, Bull. Amer. Math. Soc. 26 (1920), 370-372.

[A2] __ A lemma on systems of knotted curves, Proc. Nat. Acad. Sci. U.S.A. 9 (1923), 93-95.

[B] A. Borel, Seminar on transformation groups, Ann. of Math. Studies, No. 46, Princeton Univ. Press, Princeton, N.J., 1960.

[E] D. B. A. Epstein, The degree of a map, Proc. London Math. Soc. (3) 16 (1966), 369-383.

[F] G. K. Francis, Assembling compact Riemann surfaces with given boundary curves and branch points on the sphere, Illinois J. Math. 20 (1976), 198-217.

[He] M. Heins, Interior mapping of an orientable surface into $S^{2}$, Proc. Amer. Math. Soc. 2 (1951), 951-952.

[Hil] H. M. Hilden, Three-fold branched coverings of $S^{3}$, Amer. J. Math. 98 (1976), 989-997.

[Hir1] U. Hirsch, Uber offene Abbildungen auf die 3-Sphäre, Math. Z. 140 (1974), 203-230.

[Hir2] , Offene Abbildungen von Flächen auf die 2-Sphäre mit minimalem Defekt, Arch. Math. 27 (1976), 649-656.

[Hir3] _ On regular homotopy of branched coverings of the sphere, Manuscripta Math. 21 (1977), 293-306.

[Hud] J. F. P. Hudson, Piecewise linear topology, Benjamin, New York, 1969.

[Hur] A. Hurwitz, Uber Riemann'sche Flächen mit gegebenen Verzweigungspunkten, Math. Ann. 39 (1891), 1-60.

[Li1] W. B. R. Lickorish, Homeomorphisms of non-orientable two-manifolds, Proc. Cambridge Philos. Soc. 59 (1963), 307-317.

[Li2] _ A finite set of generators for the homeotopy group of a 2-manifold, Proc. Cambridge Philos. Soc. 60 (1964), 769-778; corrigendum, Proc. Cambridge Philos. Soc. 62 (1966), 679-681.

[Lu] J. Lüroth, Note über Verzweigungsschnitte und Querschnitte in einer Riemann'schen Fläche, Math. Ann. 4 (1871), 181-184.

[M] J. M. Montesinos, Three-manifolds as 3-fold branched covers of $S^{3}$, Quart. J. Math. Oxford Ser. (2) 27 (1976), 85-94.

[Sta] J. Stallings, On fibering certain 3-manifolds, in Topology of 3-Manifolds (M. K. Fort, ed.), Prentice-Hall, Englewood Cliffs, N. J., 1962, 95-100.

[Sto] S. Stöilow, Principes topologiques de la théorie des fonctions analytiques, Gauthier-Villars, Paris, 1938.

[T] R. Thom, Quelques propriétés globales des variétés différentiables, Comment. Math. Helv. 28 (1954), 17-85. 
[Wi] R. J. Wille, Sur la transformation intérieure d'une surface non-orientable sur le plan projéctif, Indag. Math. 56 (1953), 63-65.

[Wo] J. W. Wood, Foliations on 3-manifolds, Ann. of Math. (2) 89 (1969), 336-358.

Department of Mathematics, Cornell University, Ithaca, New Yosk 14853 (Current address of Israel Berstein)

Current address (A. L. Edmonds): Department of Mathematics, Indiana University, Bloomington, Indiana 47401 